\title{
Methanobactin reverses acute liver failure in a rat model of Wilson disease
}

\author{
Josef Lichtmannegger, ${ }^{1}$ Christin Leitzinger, ${ }^{1}$ Ralf Wimmer, ${ }^{2}$ Sabine Schmitt,, Sabine Schulz, ${ }^{1}$ Yaschar Kabiri, ${ }^{1}$ Carola Eberhagen, ${ }^{1}$ \\ Tamara Rieder, ${ }^{3}$ Dirk Janik, ${ }^{4}$ Frauke Neff, ${ }^{4}$ Beate K. Straub, ${ }^{5}$ Peter Schirmacher, ${ }^{5}$ Alan A. DiSpirito, ${ }^{6}$ Nathan Bandow, ${ }^{6}$ \\ Bipin S. Baral, ${ }^{6}$ Andrew Flatley, ${ }^{7}$ Elisabeth Kremmer, ${ }^{7}$ Gerald Denk, ${ }^{2}$ Florian P. Reiter, ${ }^{2}$ Simon Hohenester, ${ }^{2}$ \\ Friedericke Eckardt-Schupp, ${ }^{8}$ Norbert A. Dencher, ${ }^{9}$ Jerzy Adamski, ${ }^{10,11,12}$ Vanessa Sauer, ${ }^{13}$ Christoph Niemietz, ${ }^{13}$ \\ Hartmut H.J. Schmidt, ${ }^{13}$ Uta Merle, ${ }^{14}$ Daniel Nils Gotthardt, ${ }^{14}$ Guido Kroemer, ${ }^{15,16,17,18,19,20}$ Karl Heinz Weiss, ${ }^{14}$ and Hans Zischka' \\ 'Institute of Molecular Toxicology and Pharmacology, Helmholtz Center Munich, German Research Center for Environmental Health, Neuherberg, Germany. ${ }^{2}$ Liver Center Munich, Department of Medicine II, \\ Medical Center of the University of Munich (LMU), Munich, Germany. ${ }^{3}$ Institute of Toxicology and Environmental Hygiene, Technical University Munich, Munich, Germany. ${ }^{4}$ Institute of Pathology, \\ Helmholtz Center Munich, German Research Center for Environmental Health, Neuherberg, Germany. ${ }^{5}$ nnstitute of Pathology, INF 224, University Clinic Heidelberg, Heidelberg, Germany. \\ ${ }^{6}$ Roy J. Carver Department of Biochemistry, Biophysics and Molecular Biology, lowa State University, Ames, lowa, USA. Institute of Molecular Immunology, Helmholtz Center Munich, \\ German Research Center for Environmental Health, Munich, Germany. ${ }^{8}$ Institute of Radiation Biology, Helmholtz Center Munich, Cerman Research Center for Environmental Health, \\ Neuherberg, Germany. ${ }^{9}$ Department of Chemistry, Technical University Darmstadt, Darmstadt, Germany. ${ }^{10}$ Institute of Experimental Genetics, Genome Analysis Center, Helmholtz Center Munich, \\ German Research Center for Environmental Health, Neuherberg, Germany. "'Chair in Experimental Genetics, Technical University Munich, Freising-Weihenstephan, Cermany. \\ ${ }^{12}$ Cerman Center for Diabetes Research, Neuherberg, Germany. ${ }^{13}$ Clinic for Transplantation Medicine, Münster University Hospital, Münster, Cermany. ${ }^{14}$ Department of Gastroenterology, \\ Internal Medicine IV, University Hospital Heidelberg, Heidelberg, Germany. ${ }^{15}$ INSERM, U848, Villejuif, France. ${ }^{16}$ Université Paris Descartes - Paris V, Sorbonne Paris Cité, Paris, France. \\ ${ }^{17}$ Metabolomics Platform, Institut Gustave Roussy, Villejuif, France. ${ }^{18}$ Equipe 11 Labellisée Ligue contre le Cancer, Centre de Recherche des Cordeliers, Paris, France. ${ }^{19}$ Pôle de Biologie, \\ Hôpital Européen Georges Pompidou, AP-HP, Paris, France. ${ }^{20}$ Karolinska Institute, Department of Women's and Children's Health, Karolinska University Hospital, Stockholm, Sweden.
}

In Wilson disease (WD), functional loss of ATPase copper-transporting $\beta$ (ATP7B) impairs biliary copper excretion, leading to excessive copper accumulation in the liver and fulminant hepatitis. Current US Food and Drug Administration- and European Medicines Agency-approved pharmacological treatments usually fail to restore copper homeostasis in patients with WD who have progressed to acute liver failure, leaving liver transplantation as the only viable treatment option. Here, we investigated the therapeutic utility of methanobactin (MB), a peptide produced by Methylosinus trichosporium OB3b, which has an exceptionally high affinity for copper. We demonstrated that ATP7B-deficient rats recapitulate WD-associated phenotypes, including hepatic copper accumulation, liver damage, and mitochondrial impairment. Short-term treatment of these rats with MB efficiently reversed mitochondrial impairment and liver damage in the acute stages of liver copper accumulation compared with that seen in untreated ATP7B-deficient rats. This beneficial effect was associated with depletion of copper from hepatocyte mitochondria. Moreover, MB treatment prevented hepatocyte death, subsequent liver failure, and death in the rodent model. These results suggest that MB has potential as a therapeutic agent for the treatment of acute WD.

\section{Introduction}

Wilson disease (WD) is an autosomal recessively inherited disorder of copper metabolism caused by ATPase copper-transporting $\beta$ $(A T P 7 B)$ gene mutations $(1-3)$. ATP7B is a copper-transporting ATPase that mediates copper excretion into bile $(1,4)$, and its functional loss causes a major impairment of the ability of hepatocytes to maintain copper homeostasis at the cellular and

\section{Related Commentary: p. 2412}

Authorship note: J. Lichtmannegger and C. Leitzinger are co-first authors. F. Eckardt-Schupp is deceased.

Conflict of interest: During the revision phase of this manuscript, H. Zischka, J. Lichtmannegger, and Alan A. DiSpirito filed a patent application on the clinical utility of copper chelation using methanobactin ("Means and methods for treating copper-related diseases." Patent application no. EP15201070.8)

Submitted: October 22, 2015; Accepted: April 12, 2016

Reference information: / Clin Invest. 2016;126(7):2721-2735. doi:10.1172/JCI85226. systemic levels, resulting in persistent copper accumulation in the liver. This can lead, most likely as a result of spillover of liver copper, to deleterious effects on the brain and, in many cases, to chronic liver disease and also to fulminant hepatic failure $(1,5-8)$.

To restore copper homeostasis, the clinically used copper chelators D-penicillamine (D-PA) and trientine (TETA) or the candidate drug tetrathiomolybdate (TTM) are administered daily $(1,5)$. This lifelong therapy is effective if commenced before the onset of advanced hepatic or neurologic disease (6-8). The same holds true for zinc salts, which are primarily used in mild cases of WD to decrease copper absorption via the gastrointestinal tract or as copper maintenance therapy in chelator-treated WD patients $(1,5,9)$. However, in circumstances of acute liver failure - caused by delayed diagnosis, treatment failure, or rapidly developing fulminant hepatitis - death is almost certain unless liver transplantation is performed $(1,5,7,10,11)$. Poor treatment compliance may be another reason for the death 

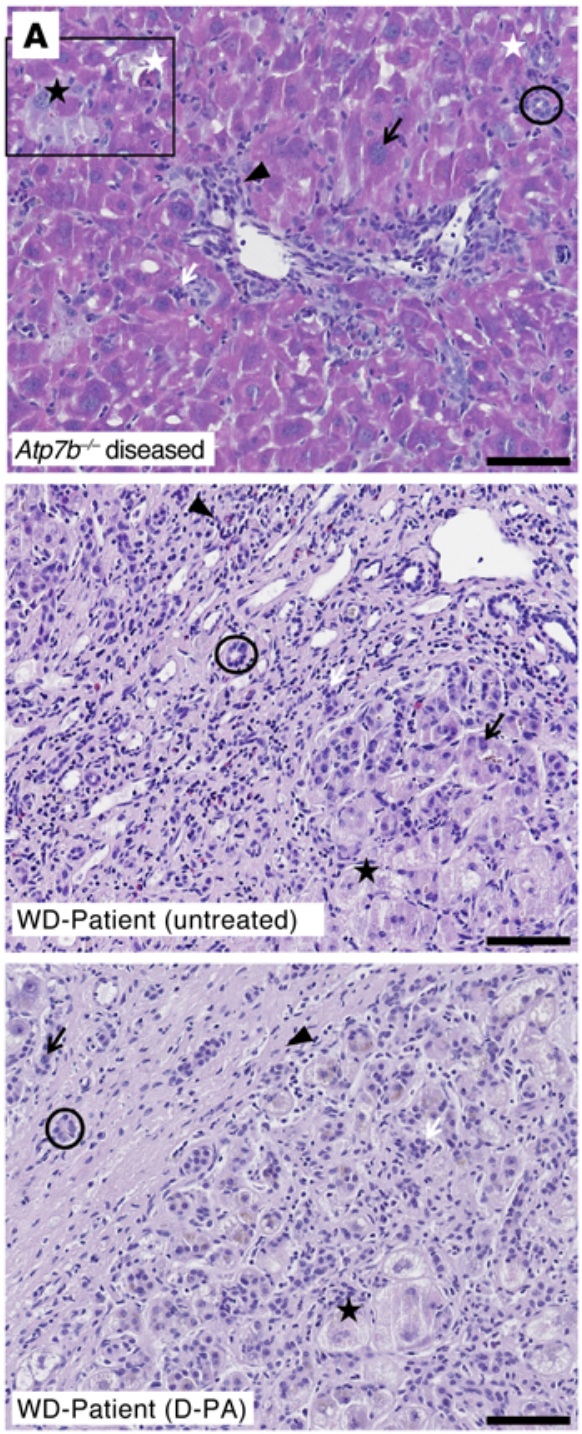
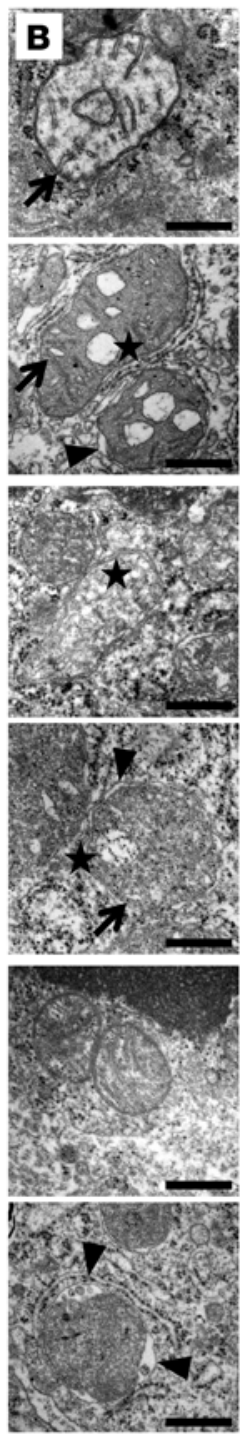

C

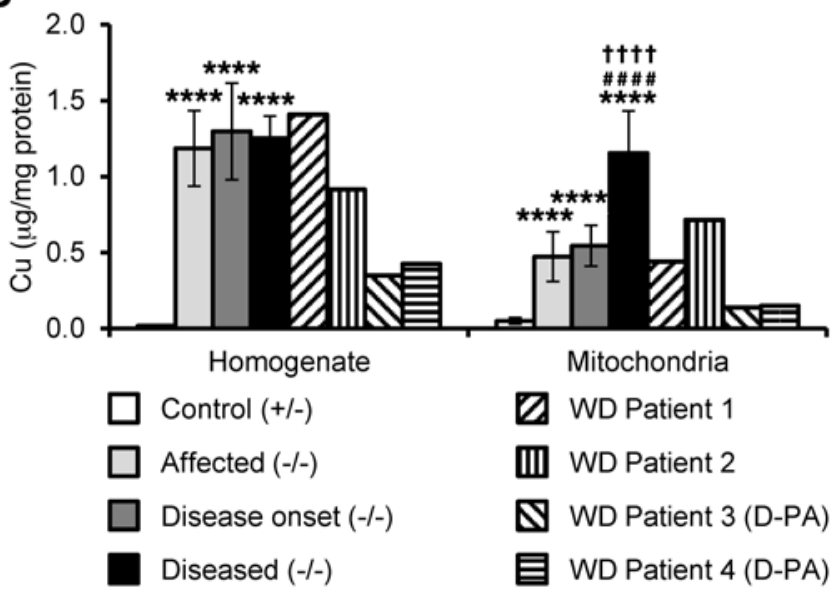

Figure 1. Liver disease in the LPP rat mirrors acute liver failure in WD patients by a devastating mitochondrial copper overload. (A) H\&E staining of liver damage in diseased $A t p 7 b^{-/-}$rats and WD patients with acute liver failure who were either untreated or D-PA treated. Tissue necrosis with resorptive inflammation as well as repair (fibrosis) were detectable (black arrowhead); proliferation of bile ducts (circle), anisokaryosis (black arrow), and several inflammatory infiltrations (white arrow) are marked. Insert shows apoptosis (white asterisk) and nodules with ballooned hepatocytes (black asterisk). Scale bars: $100 \mu \mathrm{m}$. (B) Mitochondrial structure impairments in diseased Atp76/- rats (top panels) and in WD patients with acute liver failure who were either untreated (middle panels) or D-PA treated (bottom panels). Vacuolization (asterisks) and cristae dilations (arrows), marked differences in electron densities, and separated inner and outer membranes (arrowheads) could be identified. Bottom panels: Some areas had relatively intact mitochondria (top), and others demonstrated severe structural impairments (bottom). Scale bars: $500 \mathrm{~nm}$. (C) Comparable copper burden in whole-liver homogenates and purified liver mitochondria from Atp $7 b^{-/-}$rats and untreated WD patients with acute liver failure (patients 1 and 2). Lower total copper content was detected in tissue homogenates and isolated mitochondria from the livers of WD patients who underwent D-PA treatment. Control Atp7 $b^{+/-}$rats

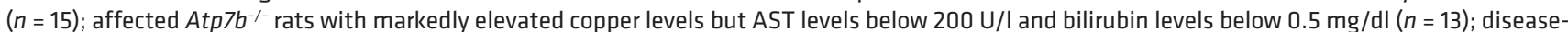
onset $A t p 7 b^{-/-}$rats with AST levels above $200 \mathrm{U} / \mathrm{l}$, bilirubin levels below $0.5 \mathrm{mg} / \mathrm{dl}(n=8)$; diseased Atp7 $\mathrm{b}^{-/-}$rats with AST levels above $200 \mathrm{U} / \mathrm{l}$ and bilirubin levels above $0.5 \mathrm{mg} / \mathrm{dl}(n=9)$. Data were outlier corrected. One-way ANOVA with Tukey's multiple comparisons test. ${ }^{* * *} P<0.0001$ versus control; \#\#\#\# $P<0.0001$ versus affected; ${ }^{\dagger+t \dagger} P<0.0001$ versus disease onset.

of patients with WD (11). All of the currently Food and Drug Administration-approved and European Medicines Agencyapproved copper chelators have adverse effects, including bone marrow toxicity, nephrotoxicity, hepatotoxicity, anemia, and triggering of autoimmune disease $(1,5,7)$. Because of the toxicity of D-PA, discontinuation of treatment is required in almost one-third of patients with WD (5).
Given these issues, there is a clear, unmet medical need for alternative and innovative WD treatments. In this regard, 3 recent reports merit special attention. First, gene therapy via adeno-associated viral vectors designed for liver-specific ATP7B expression provided long-term correction of copper metabolism in $A t p 7 b^{-1-}$ mice (12). This is an exciting new route for treating patients with $\mathrm{WD}$; however, as transgene expression upon vector injection is 


\begin{tabular}{|c|c|c|c|c|c|c|}
\hline Group (genotype) & Age (d) & Weight (g) & AST (U/I) & Bilirubin (mg/dl) & Homogenate & Mitochondria \\
\hline$F, N=15$ & $83-146$ & $170 \pm 13$ & $115 \pm 28$ & $<0.5$ & $14 \pm 2$ & $27 \pm 2$ \\
\hline $\mathrm{M}, N=9$ & $81-95$ & $281 \pm 36$ & $128 \pm 37$ & $<0.5$ & $15 \pm 1$ & $49 \pm 11$ \\
\hline$M, N=11$ & $66-93$ & $253 \pm 25$ & $151 \pm 33$ & $<0.5$ & $1,188 \pm 196$ & $464 \pm 248$ \\
\hline \multicolumn{7}{|c|}{ Disease onset (-/-) } \\
\hline $\mathrm{F}, N=4$ & $85-93$ & $150 \pm 3$ & $331 \pm 45$ & $<0.5$ & $1,385 \pm 58$ & $556 \pm 30$ \\
\hline$M, N=6$ & $80-107$ & $275 \pm 45$ & $244 \pm 55$ & $<0.5$ & $1,337 \pm 155$ & $476 \pm 159$ \\
\hline \multicolumn{7}{|l|}{ Diseased (-/-) } \\
\hline $\mathrm{M}, N=1$ & 95 & 255 & 570 & 8.0 & 1,261 & 1,602 \\
\hline
\end{tabular}

rather slow, this drawback may hamper its use in acute WD (13). A second study reported that the plant-derived decapeptide OSIP108 protected against copper-induced cell death in yeast and human HepG2 cells. Intriguingly, this positive effect was not due to a reduction of cellular copper but rather to effects on sphingolipid homeostasis (14). A third study reported hepatocyte targeting and intracellular copper chelation by specifically designed glycocyclopeptides (15). Despite this recent progress involving new therapeutic routes in WD, these studies also emphasize the unmet need for new medications that especially counteract acute liver failure in WD.

We recently described copper-driven fulminant hepatitis in LPP $A t p 7 b^{-/-}$rats that carry a homozygous $13-\mathrm{kb}$ deletion in the $A t p 7 b$ gene (hereafter referred to as $A t p 7 b^{-/-}$rats) (16-18). Because of this recessive genetic defect, heterozygous $A t p 7 b^{+/-}$rats have a normal phenotype. In $A t p 7 b^{-/-}$rats, however, accumulating liver copper results in WD-like pathology. Clinical manifestations include elevated levels of serum aspartate aminotransferase (AST) and bilirubin, rapidly followed by liver failure and death (18). The early hepatocellular phenotype in these animals is characterized by increased mitochondrial copper accumulation, provoking progressive alterations of their structure (18). A similar mitochondrial phenotype has been reported in WD patients and in other WD animal models (19-22). In Atp $7 b^{-/-}$rats, mitochondrial changes could be reversed by several weeks of treatment with copper chelators. However, this therapy was only successful when treatment was initiated before the onset of clinically manifest liver damage (18).

Here, we present the potential clinical utility of copper chelation using methanobactin (MB) to treat acute WD. MB is a posttranslationally modified peptide from the methanotrophic proteobacterium Methylosinus trichosporium OB3b, with a MW of 1,154 Da (metal free) and an exceptionally high copper affinity (23-25). MB (Supplemental Figure 2A; supplemental material available online with this article; doi:10.1172/JCI85226DS1) comprises 7 aa plus 2 chromophoric residues (termed OxaA and $\mathrm{OxaB}$ ) forming an $\mathrm{N}_{2} \mathrm{~S}_{2}$ binding site for a single copper ion (26). Methanotrophic proteobacteria have a high copper requirement for their copper-dependent methane oxidase $(27,28)$ and excrete MB to sequester extracellular copper $(25,27,28)$. MB is permeable through the bacterial outer membrane, is reinternalized across the cytoplasmic membrane via a TonB transporter (29), and delivers copper either to methane oxidases or to storage proteins like copper storage protein 1 (CSP1) (30).

Tested head-to-head with clinically available copper chelation treatments (D-PA or TETA), only MB demonstrated efficacy against acute WD in $A t p 7 b^{-/-}$rats, meaning that it was able to reverse the mitochondrial copper overload within a few days and to avoid causing the death of rats with severe signs of liver failure.

\section{Results}

Mitochondrial impairment is pathognomonic for hepatic failure in patients with WD and for liver damage in Atp $7 b^{-/-}$rats. Complete functional loss of ATP7B results in severe WD phenotypes in humans $(31,32)$. The $A t p 7 b^{-/-}$rat carries an Atp $7 b$ mutation that abolishes its hepatic copper transport activity $(16,17)$. These animals rapidly progress from a copper-burdened liver to hepatic failure and death (18). We therefore compared diseased livers from untreated patients with acute WD (who had undergone liver transplantation) with livers from $A t p 7 b^{-/-}$rats at progressive stages of the disease. In addition, livers from patients with WD who had received unsuccessful D-PA treatment before transplantation were included in this study (Figure 1).

To compare clinical stages of liver impairment, we classified rats at ages 80-100 days, when liver damage becomes apparent, into 3 groups: (a) rats affected by elevated liver copper levels, with serum AST below $200 \mathrm{U} / \mathrm{l}$ and bilirubin below $0.5 \mathrm{mg} / \mathrm{dl}$; (b) rats showing disease onset, with AST above $200 \mathrm{U} / 1$ and bilirubin below $0.5 \mathrm{mg} / \mathrm{dl}$; and (c) diseased rats, with AST above $200 \mathrm{U} / 1$ and bilirubin above $0.5 \mathrm{mg} / \mathrm{dl}$ (Table 1). 
A

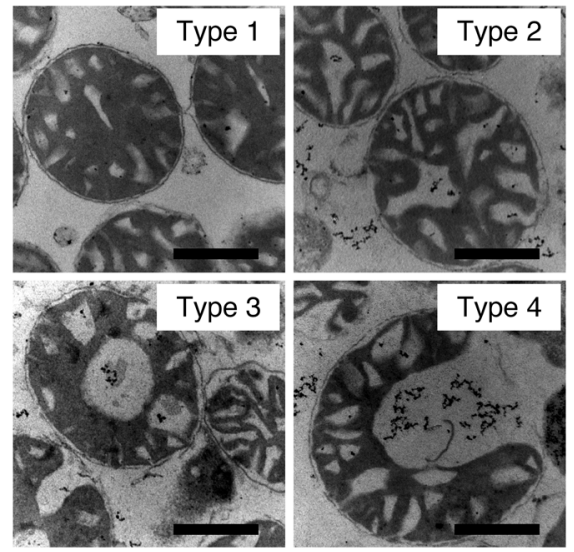

B
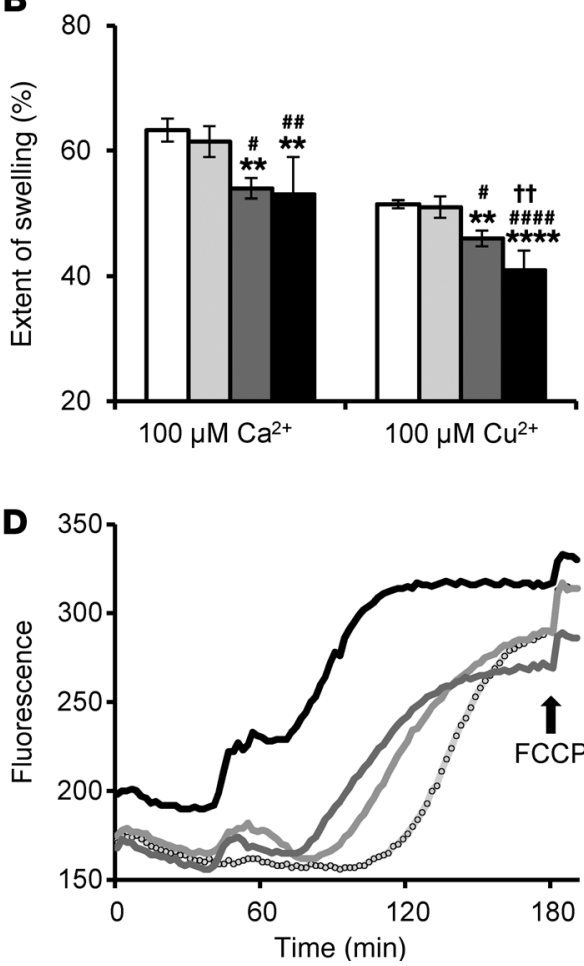
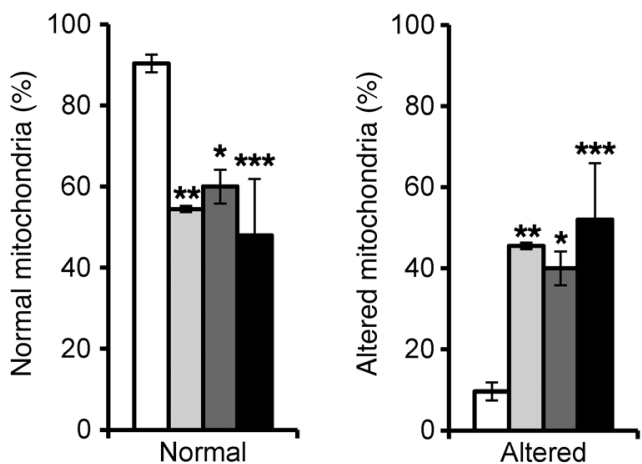

C
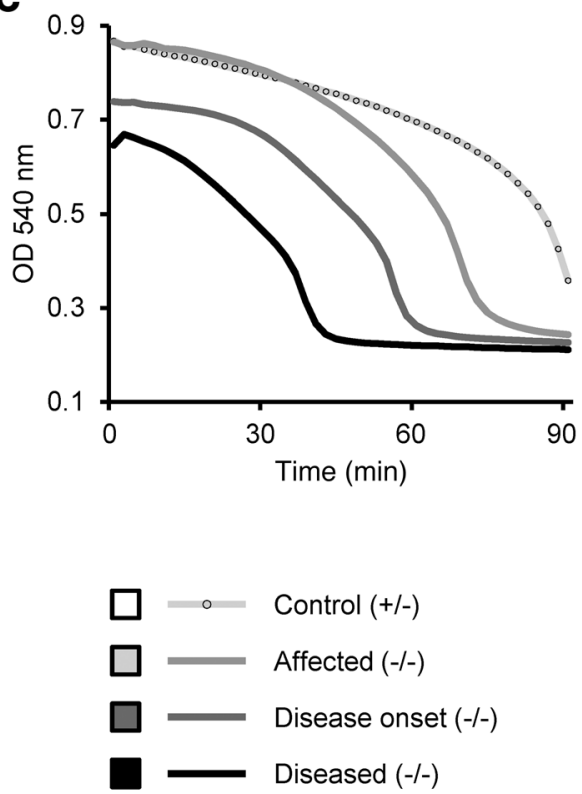

Figure 2. Increasing copper load severely attacks mitochondrial membrane integrity. (A) Progressive disease in Atp7b/- rats was paralleled by a decrease in normally structured mitochondria (Type 1 and Type 2) and an increase in structurally altered mitochondria (Type 3 and Type 4). Scale bars: 500 nm.

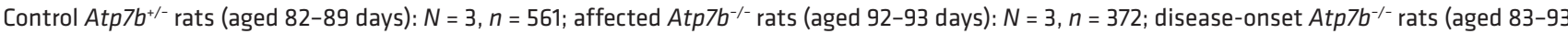
days): $N=3, n=575$; diseased Atp7 $b^{-/-}$rats (aged 104-107 days): $N=5, n=857$. (B) Upon calcium- or copper-induced MPT, isolated Atp7b/- mitochondria underwent large-amplitude swelling, which was significantly reduced in Atp $7 b^{-1-}$ mitochondria from diseased and disease-onset rats. Control Atp $7 b^{+/-}$rats:

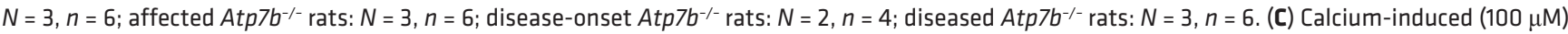
MPT could be efficiently inhibited by Cys-A $(5 \mu \mathrm{M})$. This blocking effect was severely impaired in mitochondria from diseased and disease-onset Atp $^{-1} \mathrm{~b}^{-1}$ rats. A representative comparative MPT measurement of mitochondria isolated from an $A t p 7 b^{+/-}$control rat and from Atp $7 b^{-1-}$ animals at different disease stages is depicted (quantitative analysis is shown in Supplemental Table 2). (D) Atp7b ${ }^{-/-}$mitochondria lost their membrane potential ( $\Delta \Psi$ ) at earlier time points than did control mitochondria. A representative comparative $\Delta \Psi$ measurement of mitochondria isolated from an Atp $7 b^{+/-}$control and $A t p 7 b^{-1-}$ animals at different disease stages is depicted (quantitative analysis is shown in Supplemental Table 3 ). Data in $\mathbf{A}$ and $\mathbf{B}$ were outlier corrected. $P$ values were determined by 1-way ANOVA with Tukey's multiple comparisons test. (A) ${ }^{*} P<0.05,{ }^{* *} P<0.01$, and ${ }^{* * *} P<0.001$ versus control. (B) ${ }^{* *} P<0.01$ and

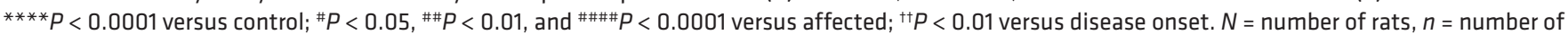
analyzed mitochondria. FCCP, carbonyl cyanide-4-(trifluoromethoxy) phenylhydrazone.

Identical tissue damage features were observed in the livers of untreated WD patients and diseased $A t p 7 b^{-/}$livers (Figure 1A). Fibrosis was observed in all WD patients' livers, and beginning fibrosis was observed in livers from diseased $A t p 7 b^{-/-}$rats (Supplemental Figure 1A). These characteristics were absent from heterozygous $A t p 7 b^{+/-}$control livers but steadily progressed in $A t p 7 b^{-1-}$ rats (Supplemental Figure 1B).

Another striking analogy between livers from patients with WD and $A t p 7 b^{-/}$rats was the structural damage of mitochondria. Transparent vacuoles of varying sizes containing amorphous but 

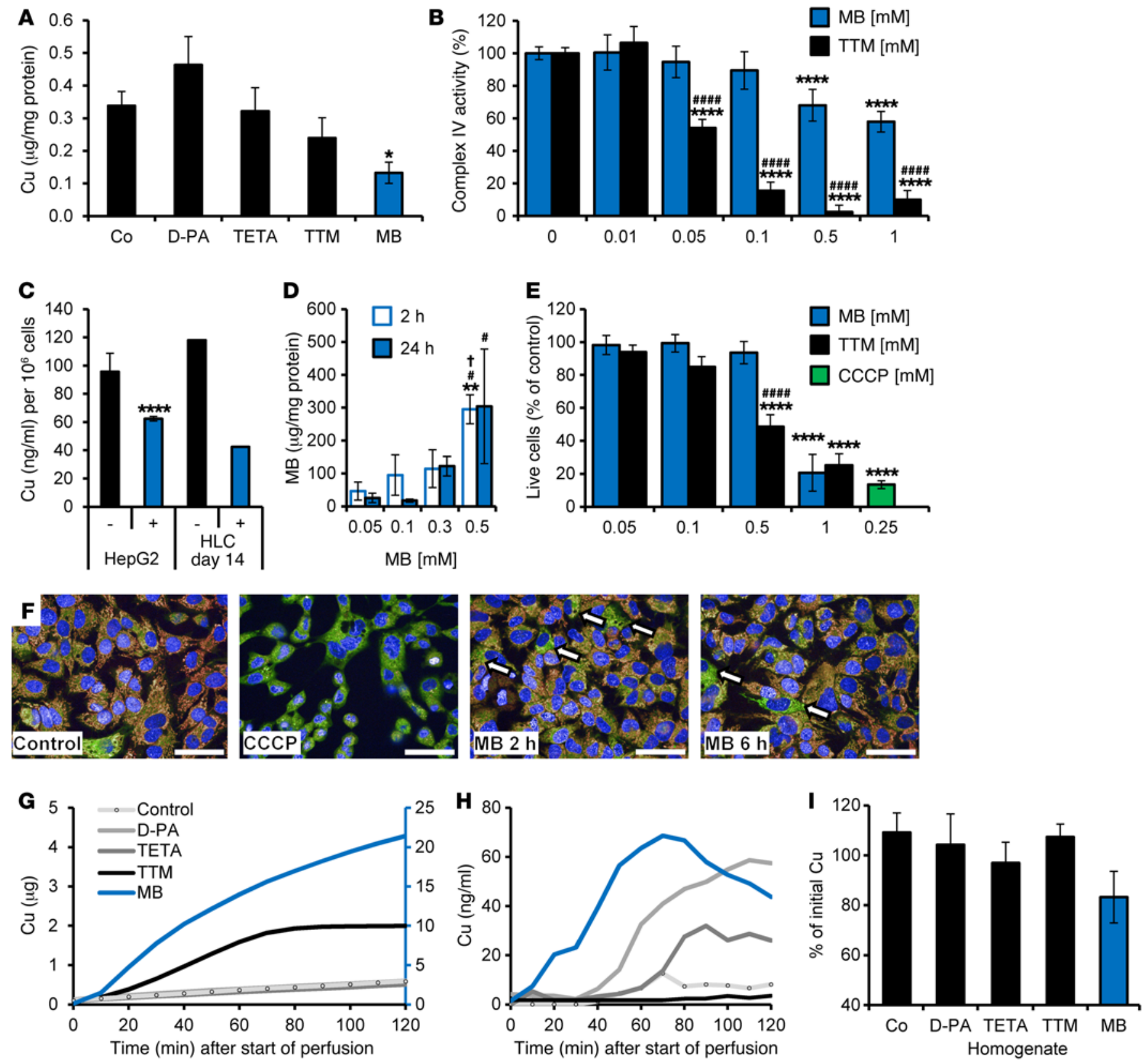

Figure 3. MB depletes copper from liver mitochondria, hepatocytes, and whole liver. (A) MB, but not D-PA or TETA, extracted copper from Atp7b ${ }^{-/-}$mitochondria (2 mM each, 30-minute incubation, $N=3$ ). (B) MB was less toxic to the mitochondrial respiratory complex IV than TTM (MB: $N=3, n=9 ;$ TTM: $N=1, n=3)$. (C) Copper-preloaded HepG2 ( $N=3)$ and WD patient-derived HLCs (1 of 2 preparations) were de-coppered by MB. (-), untreated control; ( + ), 24-hour treatment with 300-500 $\mu$ M MB. (D) Dose-dependent MB uptake into HepG2 cells at 2 and 24 hours $(N=3)$. (E) Toxicity of MB versus TTM in HepG2 (neutral red assay: $N=3, n=9)$. CCCP, positive control. (F) MB-treated ( $500 \mu M)$ HepG2 cells showed only intermediate phases of $\Delta \Psi$ loss (6 hours, $250 \mu \mathrm{M} \mathrm{CCCP).} \mathrm{Nuclei,} \mathrm{blue;} \mathrm{mitochondria} \mathrm{with} \Delta \Psi$, orange-red; mitochondria without $\Delta \Psi$, green. Arrows indicate cells with low $\Delta \Psi$ ( $N=2)$. Scale bars: $50 \mu \mathrm{m}$. (G) Cumulative copper excretion into bile following a 2-hour Atp7 $\mathrm{b}^{-/-}$liver perfusion. Only MB (0.7 $\left.\mu \mathrm{M}\right)$ forced high copper amounts into bile compared with TTM $(0.8 \mu \mathrm{M})$, D-PA $(2.2 \mu \mathrm{M})$, and TETA $(1.8 \mu \mathrm{M}) . N=3$. Note the different scales for MB (right, blue axis) and buffer control, D-PA, TETA, and TTM (left, black axis). (H) All chelators except TTM transported copper to the perfusate (conditions as in $\mathbf{G}) . N=2$. (I) Only MB markedly reduced liver copper levels during Atp7 $b^{-/-}$liver perfusion, but not D-PA, TETA, or TTM (conditions as in G). $N=3$. One-way ANOVA with Tukey's multiple comparisons test (A, B, D, E, and I); unpaired $t$ test with Welch's correction (C). ${ }^{*} P<0.05$ and ${ }^{* * *} P<0.0001$ versus control $(\mathbf{A}-\mathbf{C}$ and $\mathbf{E})$; $\# \# \# \#<0.0001$ versus respective $\mathrm{MB}$ concentration (B and $\mathbf{E}$ ). ${ }^{* *} P<0.01$ versus $0.05 \mathrm{mM} \mathrm{MB} ;{ }^{*} P<0.05$ versus $0.1 \mathrm{mM} \mathrm{MB}$; and ${ }^{\dagger} P<0.05$ versus $0.3 \mathrm{mM}$ MB (D). Co, buffer-treated control.

also electron-dense material, separated inner and outer membranes, marked differences in electron densities, and cristae dilations (Figure 1B and Supplemental Figure 1C) were observed as the typical WD mitochondrial phenotype (19). Importantly, we found highly comparable copper levels in liver homogenate and mitochondria obtained from diseased $A t p 7 b^{-/}$rats and untreated WD patients' livers (Figure 1C). However, lower copper content was present in the homogenate from the explanted livers and iso- 

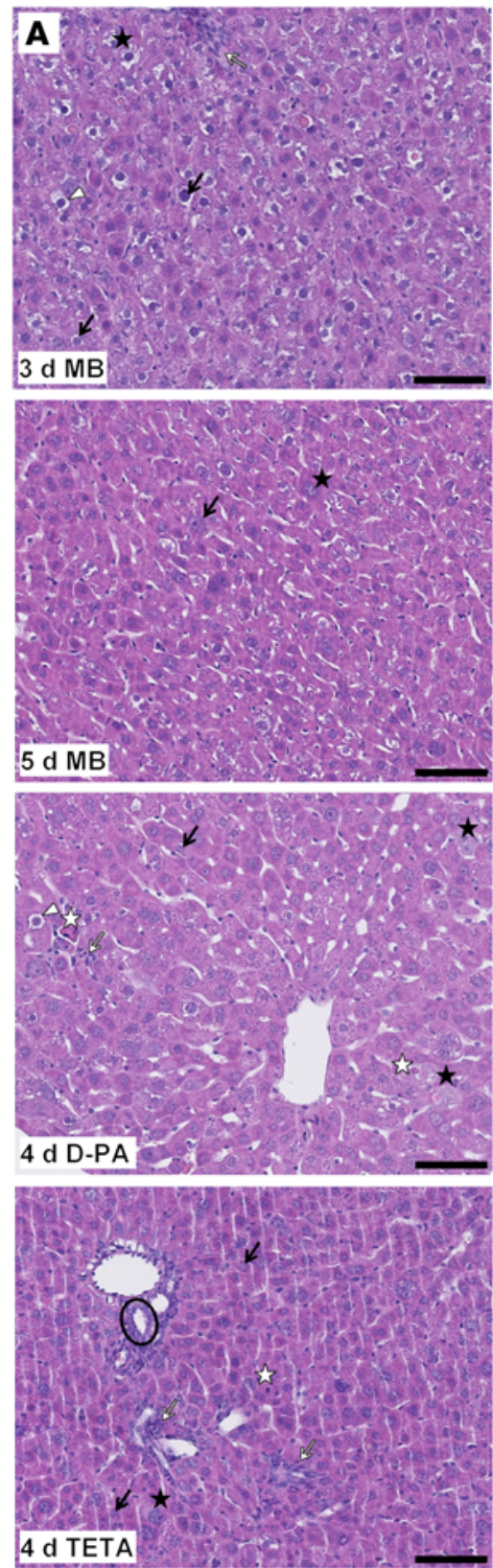

$\mathbf{B}$
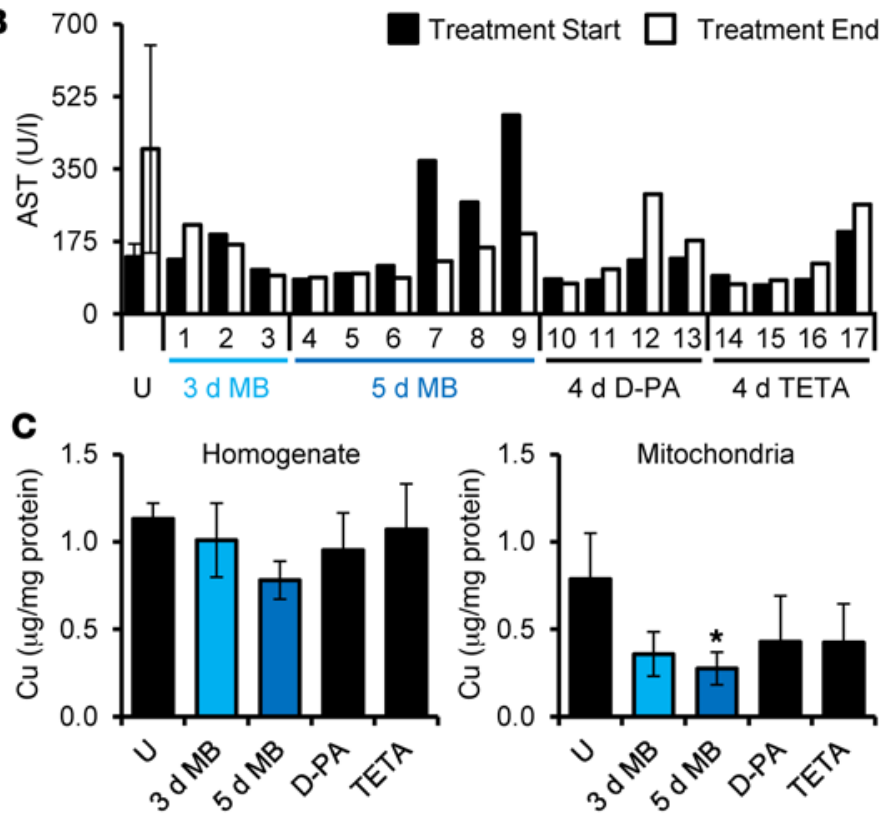

D
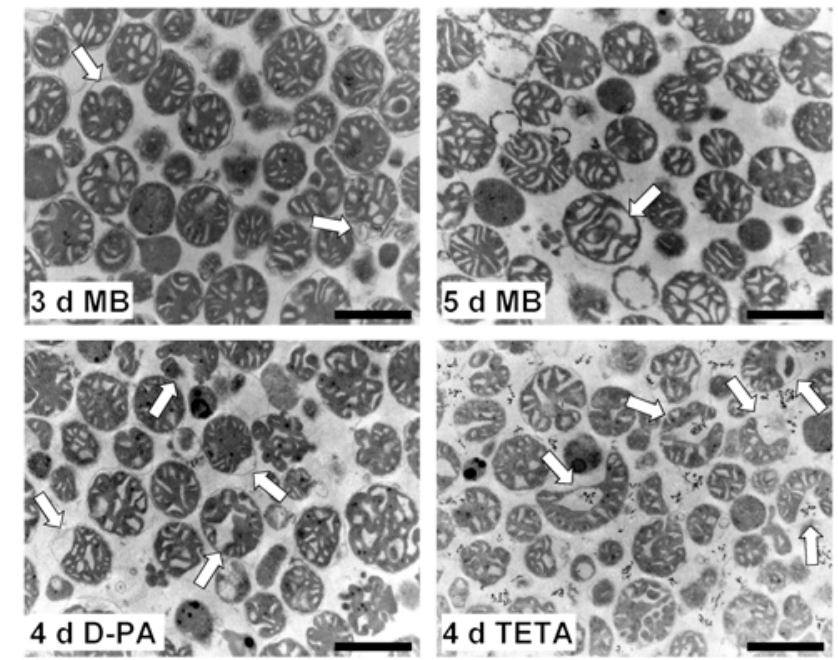

E
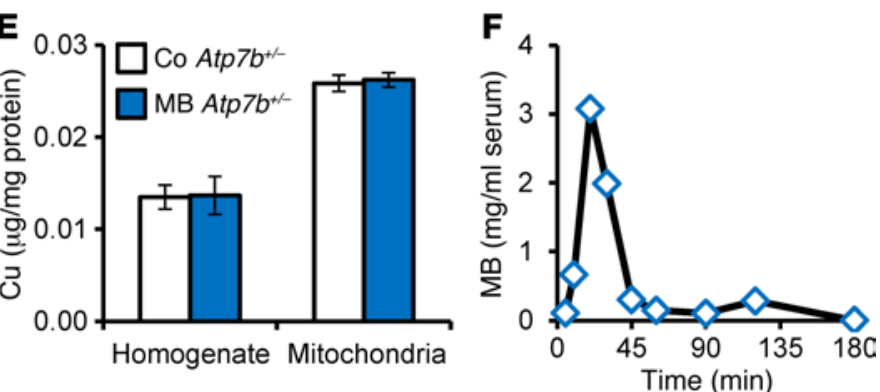

Figure 4. Acute liver failure is efficiently avoided by a short-term in vivo treatment with MB. (A) Overt liver damage was reduced in MB-treated but not D-PA- or TETA-treated Atp $7 b^{-/-}$livers. Scale bars: $100 \mu \mathrm{m} ; \mathrm{H} \&$ E staining (symbols as in Figure 1 and Supplemental Figure 1). Daily doses were: $150 \mathrm{mg}(130 \mu \mathrm{mol}) \mathrm{MB} / \mathrm{kg}$ BW; $100 \mathrm{mg}(540 \mu \mathrm{mol}) \mathrm{D}-\mathrm{PA} / \mathrm{kg} \mathrm{BW}$; and $480 \mathrm{mg}(2,190 \mu \mathrm{mol})$ TETA/kg BW. Three-day MB treatment: 2 experiments; 5-day MB treatment: 5 experiments; D-PA and TETA treatments: 4 experiments. (B) AST values decreased in 2 of 3 and 4 of 6 Atp $7 b^{-1-}$ rats treated for 3 or 5 days with MB, respectively, but not in untreated $\operatorname{Atp}^{-1-}(N=6$ [3 affected and 3 diseased]) or short-term D-PA- or TETA-treated rats ( $N=6$ [3 affected and 3 diseased]). Treatment started in rats at $82-90$ days of age. (C) Mild reduction of whole-liver and significant reduction of mitochondrial copper level in short-term MB-treated rats (3-day MB treatment, $N=3$ [2 affected and 1 disease onset], aged 88-89 days; 5-day MB treatment, $N=5$ [5 affected], aged 89-95 days) but not in untreated rats ( $N=4$ [2 affected, 2 diseased], aged 90-91 days) or D-PA- or TETA-treated rats ( $N=4$ [3 affected, 1 disease onset], aged 86-89 days). One-way ANOVA with Tukey's multiple comparisons test. ${ }^{*} P<0.05$ versus untreated controls. (D) Massively reduced numbers in mitochondria with severely impaired structure (type 4 , arrows) were isolated from MB-treated rats but not from untreated (Figure 2A) or D-PA- or TETA-treated Atp7b ${ }^{-1-}$ rats (quantification in Supplemental Figure $4 \mathrm{~A}$ ). Scale bars: $1 \mu \mathrm{m}$. (E) Treatment of $A t p 7 b^{+/-}$control rats with MB (i.p. once daily on 2 consecutive days; $N=4$ ) did not change whole-liver or mitochondrial copper levels. Untreated control, $N=3$. (F) Upon i.p. injection, MB was only detectable in the serum for half an hour, indicating a very short systemic residence time $(n=2)$. $U$, untreated. 
lated mitochondria of the D-PA-pretreated WD patients (Figure 1C). This coincided with more heterogeneous impairment of the mitochondrial structure (Figure 1B), which probably results from zonal heterogeneities originating from massive fibrosis within these livers (Figure 1A).

Increasing copper load impairs mitochondrial membrane integrity and function. Mitochondrial copper content progressively increased with disease state in livers from $A t p 7 b^{-/-}$rats (Figure $1 \mathrm{C}$ and Table 1). This was paralleled by increasingly severe membrane deficits, as demonstrated directly at the level of freshly isolated mitochondria (Figure 2) and described as follows: (a) a drastic decrease in structurally normal rat liver mitochondria (types 1 and 2) from $A t p 7 b^{-/-}$livers compared with control livers and a corresponding increase in the number of structurally altered organelles (types 3 and 4, Figure 2A); (b) a significant alteration of the mitochondrial membrane "fluidity" at the polar head groups of the membrane lipid-water interface (TMA-DPH), but not at the membrane inner lipid phase (DPH) (Supplemental Table 1), as revealed by membrane polarization measurements with the fluorophores 1,6-diphenyl-1,3,5-hexatriene (DPH) and trimethylammonium-DPH (TMA-DPH) $(33,34)$; (c) a large-amplitude swelling of control mitochondria upon induction of the mitochondrial permeability transition (MPT) $(18,35)$ by either calcium or copper that was significantly reduced in mitochondria from diseased and disease-onset $A t p 7 b^{-/-}$rats (Figure 2B); (d) a significant impairment of the capacity of Cys-A to block calcium-induced MPT in $A t p 7 b^{-/-}$versus control mitochondria (Figure 2C and Supplemental Table 2); (e) a jeopardization of the time stability of the inner mitochondrial transmembrane potential $(\Delta \Psi)$ and loss of $\Delta \Psi$ in $A t p 7 b^{-/-}$mitochondria at earlier time points compared with control mitochondria (Figure 2D and Supplemental Table 3); and (f) a progressive and significant impairment of the ATP production capacity of $A t p 7 b^{-/}$mitochondria (Supplemental Figure 4F).

The bacterial peptide $M B$ rapidly depletes accumulated mitochondrial copper. We compared the capability of MB with that of the copper chelators D-PA, TETA, and TTM to remove copper from freshly isolated $A t p 7 b^{-/}$mitochondria. While D-PA and TETA were without effect, TTM showed a tendency to remove copper, and $\mathrm{MB}$ significantly decreased the copper levels associated with $A t p 7 b^{-/-}$mitochondria (Figure 3A). We obtained similar results with mitochondria from WT rats artificially preloaded with copper (Supplemental Figure 2, B and C). Furthermore, MB was significantly less toxic than TTM, as shown by the impaired vital copper-dependent mitochondrial respiratory complex IV (Figure 3B and Supplemental Figure 2D).

$M B$ efficiently de-coppers hepatocytes with low cell toxicity. At the cellular level, overnight MB treatments significantly reduced cellular copper levels in HepG2 cells that were either copper preloaded at concentrations that showed no toxicity (Figure 3C and Supplemental Figure 2F) or in HepG2 cells that had a normal copper load (Supplemental Figure 2E). Moreover, in an attempt to test the efficacy of MB on samples from patients with WD, we reprogrammed urinary epithelial cells from these patients into induced pluripotent stem cells (iPSCs) that differentiated into hepatocytelike cells (HLCs) (Supplemental Figure 2, G-J) and found comparable copper depletion levels in these HLCs following treatment with MB (Figure 3C).
Using a specific $\mathrm{MB} \mathrm{mAb}$, we found that $\mathrm{MB}$ was taken up dose dependently into HepG2 cells (Figure 3D) and that cytotoxicity occurred only at millimolar MB concentrations (Figure $3 \mathrm{E})$. At the mitochondrial level, MB concentrations of $500 \mu \mathrm{M}$ reduced the mitochondrial membrane potential only partially (Figure 3F). Thus, MB efficiently de-coppers hepatocytes, without major toxic side effects.

$M B$ directs liver copper into bile. We further investigated the copper-removing efficiency of MB at the whole-organ level (Figure 3, G-I, Supplemental Figure 3). During a 2-hour perfusion of $A t p 7 b^{-/-}$ livers, MB released 10-fold greater amounts of copper into bile than did TTM (Figure 3G and Supplemental Figure 3B), the major physiological copper excretion route (36). D-PA and TETA did not provoke any detectable release of copper into bile (Figure 3G). All chelators, except TTM, caused an increased presence of copper in the perfusate (Figure $3 \mathrm{H}$ ), which may be linked to the ability of TTM to precipitate copper intracellularly (37). The release of copper into the perfusate was partly due to hepatocellular death, as the cellular damage marker lactate dehydrogenase (LDH) paralleled the copper release curves (Supplemental Figure 3C). Of note, only MB treatment had already markedly reduced the $A t p 7 b^{-/-}$liver copper levels after 2 hours of perfusion (Figure 3I).

Short-term $M B$ application reverses liver damage in vivo. We assessed the efficiency of short-term MB treatment in $A t p 7 b^{-1-}$ rats at the age of liver disease onset (85-90 days). Animals received either MB (i.p.) for 3 or 5 days or the clinically used copper chelators D-PA or TETA (administered orally for 4 days).

$\mathrm{MB}$ administration strongly reduced the histopathological damage markers in $A t p 7 b^{-/-}$livers (Figure 4A), in contrast to that observed with D-PA or TETA treatments. The latter 2 chelators were unable to circumvent the increase in serum AST levels (indicative of progressive liver damage, Figure 4B). In contrast, in 6 of $9 \mathrm{MB}$-treated $\mathrm{Atp} 7 \mathrm{~b}^{-/-}$rats, AST levels decreased (Figure 4B), and the animals regained BW (Table 2). Importantly, after 5 days of MB treatment, $2 A t p 7 b^{-/}$animals with onset disease and 1 diseased $A t p 7 b^{-/-}$rat were rescued from liver dysfunction (AST $<200 \mathrm{U} / 1$, Figure $4 \mathrm{~B}$ and Table 2).

In comparison with untreated, age-matched $A t p 7 b^{-/-}$rats and in contrast to D-PA or TETA treatments, MB induced a marked reduction in total liver copper levels, which was especially significant in the mitochondrial compartment (Figure 4C). This mitochondrial de-coppering was paralleled by ultrastructural and biochemical restoration of $A t p 7 b^{-/-}$mitochondria (Figure 4D and Supplemental Figure 4F). Severely impaired mitochondria (type 4, Figure 2A) were almost absent in isolates from MB-treated $A t p 7 b^{-/-}$rats, but not in isolates from D-PA- or TETA-treated animals (Figure 4D and Supplemental Figure 4A). Importantly, the impaired ATP production capacity of $A t p 7 b^{-/}$mitochondria was fully restored by $\mathrm{MB}$, a result that was significantly different from that observed with D-PA or TETA treatments, which were without therapeutic effect (Supplemental Figure 4F).

Toward a new WD treatment regimen using MB. From a clinical perspective, we initiated experiments to scrutinize the potential therapeutic use of $\mathrm{MB}$.

First, concerning drug safety, 4 heterozygous $A t p 7 b^{+-}$rats were treated i.p. with MB on 2 consecutive days. We observed no signs of toxicity, as BW, serum AST levels, and bilirubin values 
Table 2. Short-term treatments with 5× MB (once daily, i.p. or i.v.) or with 16× MB (twice daily, i.p.) reduce the mitochondrial copper load

\begin{tabular}{|c|c|c|c|c|c|c|c|c|c|c|}
\hline \multirow[t]{2}{*}{ Treatment (genotype) } & \multirow[t]{2}{*}{ Age (d) } & \multicolumn{2}{|c|}{ Weight (g) } & \multicolumn{2}{|c|}{ AST (U/I) } & \multicolumn{2}{|c|}{ Bilirubin (mg/dl) } & \multicolumn{2}{|c|}{ Cu (ng/mg protein) } & \multirow{2}{*}{$\begin{array}{c}\text { Mitochondrial } \\
\text { Cu depletion }{ }^{\mathrm{A}}(\%)\end{array}$} \\
\hline & & Start & End & Start & End & Start & End & Homogenate & Mitochondria & \\
\hline \multicolumn{11}{|l|}{ MB 2× i.p. (+/-) } \\
\hline $\mathrm{F}$, rat 1 & 89 & 188 & 181 & - & 108 & - & $<0.5$ & 13 & 26 & 2.6 \\
\hline F, rat 2 & 89 & 170 & 171 & - & 123 & - & $<0.5$ & 16 & 27 & 0.5 \\
\hline F, rat 3 & 161 & 209 & 212 & - & 139 & - & $<0.5$ & 11 & 25 & 7.1 \\
\hline $\mathrm{F}$, rat $1(\mathrm{~A})$ & 89 & 144 & 160 & 131 & 215 & $<0.5$ & $<0.5$ & 1,307 & 525 & 5.5 \\
\hline$M$, rat $2(A)$ & 88 & 261 & 261 & 192 & 169 & $<0.5$ & $<0.5$ & 881 & 335 & 27.8 \\
\hline$M$, rat $3(A)$ & 88 & 259 & 260 & 106 & 93 & $<0.5$ & $<0.5$ & 840 & 215 & 53.7 \\
\hline \multicolumn{11}{|l|}{ MB 5× i.p. (-I-) } \\
\hline $\mathrm{F}$, rat $1(\mathrm{~A})$ & 91 & 157 & 154 & 116 & 87 & $<0.5$ & $<0.5$ & - & 205 & 50.7 \\
\hline$M$, rat $6(A)$ & 89 & 258 & 269 & 97 & 97 & $<0.5$ & $<0.5$ & 600 & 177 & 61.9 \\
\hline \multicolumn{11}{|l|}{ MB 5× i.v. (-I-) } \\
\hline $\mathrm{F}$, rat $1(\mathrm{D})$ & 92 & 135 & 143 & 295 & 155 & 1.5 & $<0.5$ & 928 & 532 & 20.6 \\
\hline F, rat 2 (DO) & 92 & 150 & 161 & 70 & 107 & 1.2 & $<0.5$ & 1265 & 391 & 29.7 \\
\hline M, rat 3 (DO) & 81 & 234 & 240 & 97 & 98 & 1.6 & $<0.5$ & 721 & 173 & 63.7 \\
\hline \multicolumn{11}{|l|}{ MB 16× i.p. (-/-) } \\
\hline $\mathrm{F}, \operatorname{rat} 1$ (D) & 89 & 148 & 155 & 365 & 182 & 1.0 & $<0.5$ & 455 & 241 & 64.0 \\
\hline F, rat 2 (D) & 89 & 144 & 160 & 570 & 178 & 0.9 & $<0.5$ & 315 & 218 & 67.4 \\
\hline F, rat $3\left(M_{0}\right)$ & 90 & 120 & 155 & 610 & 122 & 15.5 & $<0.5$ & 220 & 203 & 85.5 \\
\hline F, rat 4 (DO) & 90 & 143 & 163 & 273 & 131 & $<0.5$ & $<0.5$ & 513 & 206 & 62.9 \\
\hline
\end{tabular}

AMitochondrial copper depletion relating to the respective untreated group (Table 1). A, affected; DO, disease onset; D, diseased; Mo, moribund.

remained within the physiological range (Table 2). Furthermore, no changes in total liver copper levels or in the mitochondrial compartment were measured compared with untreated $A t p 7 b^{+/-}$controls (Figure 4E). Similarly, ATP production of mitochondria from MB-treated and untreated heterozygous $A t p 7 b^{+/-}$rats was highly comparable (Supplemental Figure 4F). Moreover, upon i.p. injection, $\mathrm{MB}$ was detectable in the serum for only 45 minutes, indicating a very short systemic residence time (Figure $4 \mathrm{~F}$ ). Thus, $\mathrm{MB}$ efficiently removed excessive liver copper in homozygous Atp $7 b^{-/-}$ rats, but such treatments were without obvious signs of toxicity in $A t p 7 b^{-/-}$rats or heterozygous $A t p 7 b^{+/-}$control rats.

Second, with respect to alternative MB application routes, we found that either i.p. or i.v. injection could be used (Table 2). For i.v. injections, $3 \mathrm{Atp} 7 \mathrm{~b}^{-/-}$rats were catheterized via the femoral vein. After a 3-day recovery period, animals received daily $\mathrm{MB}$ doses on 5 consecutive days. All animals regained BW, and in rats with elevated AST or bilirubin, their levels returned to normal. Moreover, we observed a profound reduction in copper content at the whole-liver and purified mitochondrial levels (Table 2).

Third, we assessed the length of time that a short-term MB treatment postpones the onset of acute liver failure. Three Atp $7 b^{-/}$ rats were treated with MB for 5 days, and MB was discontinued during the subsequent observation phase. Starting with the MB treatment, zinc-enriched food $(1,000 \mathrm{ppm})$ was given, as zinc is a clinically relevant copper maintenance therapy in WD (9). All MB-treated animals showed restoration of normal serum AST levels that lasted for at least 2 weeks, after which AST levels rose again (Figure 5A). At the time of analysis, 1 animal was still healthy, and 2 animals manifested different stages of liver disease (Figure 5, A and C). The degree of liver damage correlated with mitochondrial, but not whole-liver, copper levels (Figure 5B), as well as with structural (Figure 5D) and functional defects in mitochondria (Supplemental Figure 4B).

Fourth, we conducted a first test involving replacement of daily chelation therapy by a regimen consisting of intense treatment cycles interrupted by observation periods (Table 3). Five $A t p 7 b^{-/-}$rats as well as 5 age- and sex-matched $A t p 7 b^{+-}$controls (untreated) were included, and pairs of rats were sacrificed on experimental days $1,8,29,36$, and 85, respectively. On experimental day 1 , all animals were healthy, with the sacrificed $A t p 7 b^{-/-}$rat showing a pronounced liver and mitochondrial copper load and slightly impaired mitochondrial function (87\% ATP production capacity) compared with its $A t p 7 b^{+/-}$control (pair 1). The 4 remaining $A t p 7 b^{-/-}$rats were subjected to the first treatment cycle consisting of 3 daily i.p. MB injections for 5 days. All animals remained healthy, and this resulted in a $40 \%$ reduction in the copper load on experimental day 8 (pair 2), which increased to starting levels after an additional 3 weeks of observation (pair 3, day 29). Fol- 
A
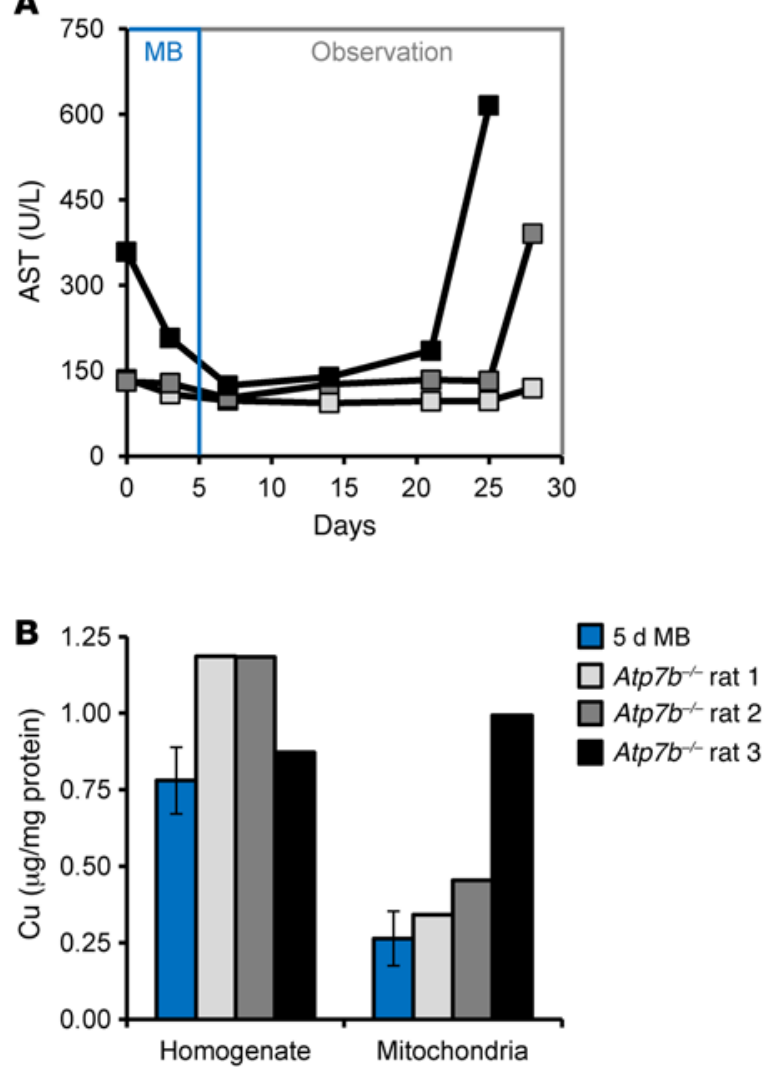

C
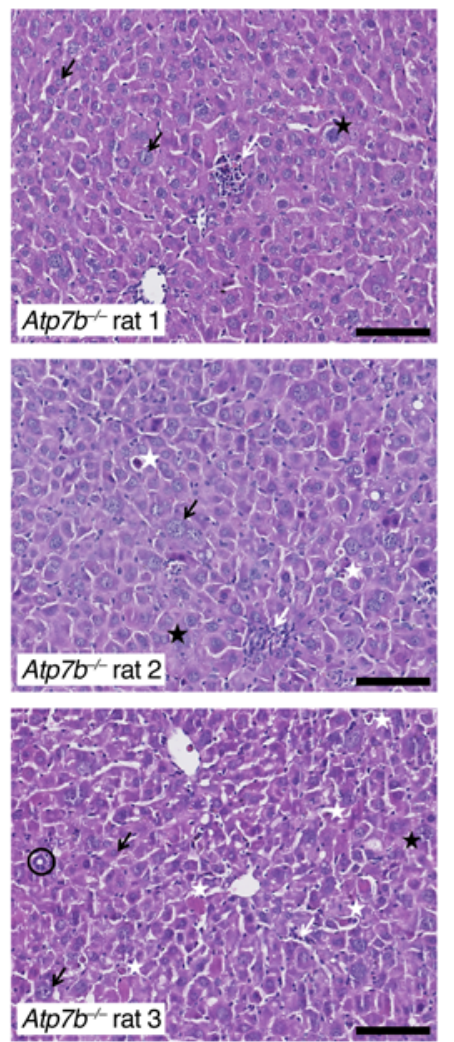

D
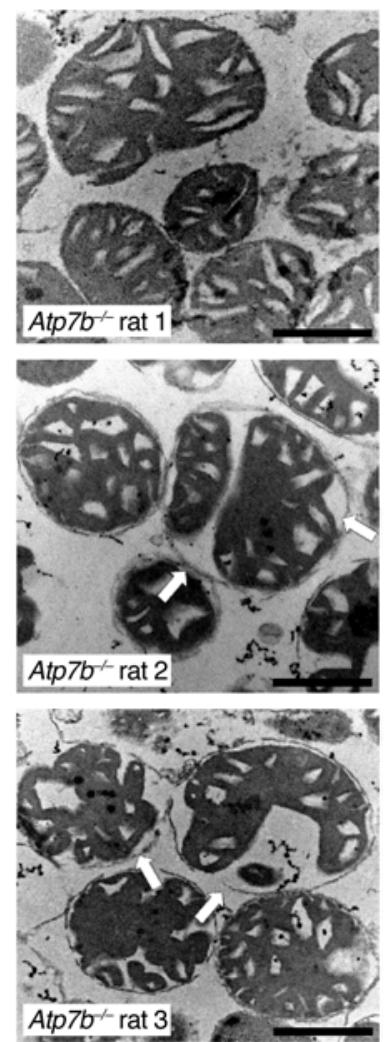

Figure 5. Short-term MB treatments postpone acute liver failure. (A) Short-term ( 5 days) MB-treated Atp7b ${ }^{-/}$rats (animal age at start of treatment: 84-85 days) remained healthy for at least 2 weeks, after which serum AST and bilirubin (not shown) levels rose again. At the time of analysis, 1 animal (Atp $7 b^{-/-}$rat 1 ) was still healthy, and 2 animals (Atp7b/- rats 2 and 3 ) were diseased. (B-D) In the order of Atp7b/- rats 1 to 3 , respectively, mitochondrial copper content was elevated but not the whole-liver copper content (B), the frequency of the typical histological features of overt liver damage increased (C, symbols as in Figure 1 and Supplemental Figure 1), and the severity of mitochondrial structure impairments increased (D, arrows). Scale bars: $100 \mu \mathrm{m}$ (C) and $500 \mathrm{~nm}$ (D)

lowing the second treatment cycle, copper loads decreased again, but were now down to $25 \%$ of the starting values, resulting in an unprecedented low copper load in $A t p 7 b^{-/}$mitochondria (pair 4, day 36$)$. This $75 \%$ de-coppering efficiency was associated with a subsequent observation period of an additional 7 weeks, during which the remaining $A t p 7 b^{-/}$rat stayed healthy. This corresponds to a doubling of the age when untreated $A t p 7 b^{-/}$rats normally become diseased. On experimental day 85 (pair 5), we found that liver and mitochondrial copper loads were similar to those of the $A t p 7 b^{-/}$rat before the start of treatment and that mitochondrial function was impaired (65\% of the ATP production capacity of the $A t p 7 b^{+/-}$control).

$M B$ for the treatment of acute liver failure. We assessed the capacity of MB to rescue diseased $A t p 7 b^{-/}$rats by an "acute rescue regimen" consisting of 2 daily MB injections for 1 week (16 i.p. injections in total, Table 2). Four $A t p 7 b^{-1-}$ rats with strongly elevated AST levels were treated. All animals survived, regained BW, and presented with normal serum AST and bilirubin levels and exceptionally low copper values at the end of the treatment regimen (Table 2). This powerful therapeutic effect was best exemplified by the case of animal 3. Diseased $A t p 7 b^{-/}$rats presenting with progressive BW loss and bilirubin levels greater than $8 \mathrm{mg} /$ $\mathrm{dl}$ (Table 1) must be considered moribund, as such animals usually die within a few days. In contrast, following the "acute rescue regimen," animal 3 regained $29 \%$ of its BW, had a drastic reduction of AST and bilirubin to normal levels, and hepatic copper depletion associated with massive structural and functional mitochondrial recovery (Table 2 and Supplemental Figure 4, D-F).

\section{Discussion}

Mitochondrial copper overload occurs to a similar extent in WD patients and $A t p 7 b^{-/}$rats (Figure 1). This overload causes mitochondrial impairments (Figure 2) that lead to liver damage (Figures 1 and 5 and Supplemental Figures 1 and 4). Failure to respond to established treatments in human WD has been associated with a failure to deplete mitochondrial copper stores (38). Thus, reversal of mitochondrial copper overload seems to be a prerequisite for successful treatment. Clinically used copper chelators slowly deplete copper stores, are ineffective in some patients with chronic $\mathrm{WD}$, and fail to rescue many patients with acute, fulminant WDinduced hepatitis and liver failure $(1,5,10)$. Here, we show that MB rapidly and effectively depleted excess hepatic copper stores at mitochondrial, cellular, and organ levels (Figure 3 and Supplemental Figures 2 and 3). In vivo, MB reversed WD-associated hepatitis and was effective even when applied in acute, fulminant liver disease (Figures 4 and 5; Supplemental Figure 4; and Table 2). 
Table 3. A new treatment regimen consisting of intense MB treatment cycles interrupted by drug-free observation periods

\begin{tabular}{|c|c|c|c|c|c|c|c|c|c|c|}
\hline \multirow{2}{*}{$\begin{array}{l}\text { Rat pair } \\
\text { Atp7b genotype }\end{array}$} & \multicolumn{2}{|c|}{1} & \multicolumn{2}{|c|}{2} & \multicolumn{2}{|c|}{3} & \multicolumn{2}{|c|}{4} & \multicolumn{2}{|c|}{5} \\
\hline & $+/-$ & $-1-$ & $+/-$ & $-1-$ & $+/-$ & $-1-$ & $+/-$ & $-1-$ & $+/-$ & $-1-$ \\
\hline Age (d) & 82 & 82 & 89 & 89 & 109 & 109 & 117 & 116 & 166 & 166 \\
\hline Sex & $\mathrm{F}$ & $\mathrm{F}$ & $\mathrm{F}$ & $\mathrm{F}$ & $\mathrm{F}$ & $\mathrm{F}$ & $M$ & $M$ & $M$ & $M$ \\
\hline AST (U/I) Start & - & - & - & 88 & - & 110 & - & 94 & - & 88 \\
\hline AST (U/I) End & 118 & 113 & 93 & 88 & 78 & 86 & 90 & 83 & 137 & 109 \\
\hline \multicolumn{11}{|l|}{ Cu ( $\mu \mathrm{g} / \mathrm{mg}$ protein) } \\
\hline Homogenate & 0.013 & 0.917 & 0.014 & 0.593 & 0.012 & 1.001 & 0.013 & 0.225 & 0.014 & 1.019 \\
\hline Mitochondria & 0.028 & 0.296 & 0.027 & 0.167 & 0.027 & 0.267 & 0.028 & 0.067 & 0.023 & 0.266 \\
\hline
\end{tabular}

An unprecedented low $A t p 7 b^{-/-}$mitochondrial copper load was found after the second treatment cycle and was associated with a disease-free state until experimental day 85, which corresponded to a doubling of the age when untreated $A t p 7 b^{-1-}$ rats become diseased. Five Atp $7 b^{-1-}$ rats and five age- and sex-matched $\mathrm{Atp} \mathrm{b}^{+/-}$controls (untreated) were compared pairwise on experimental days 1, 8, 29, 36, and 85, respectively. A pronounced copper load was found in $A t p 7 b^{-/-}$rat 1 on experimental day 1, and the remaining 4 Atp $7 b^{-/-}$rats were treated with MB (3x/day i.p., 5 days). This resulted in a $40 \%$ reduction in copper load in $A t p 7 b^{-/-}$rat 2 by experimental day 8 , a level that increased after 3 weeks of observation in Atp7 $b^{-/-}$rat 3 . An unprecedentedly low mitochondrial copper load was found in $A t p 7 b^{-/-}$rat 4 after the second treatment cycle (days 29-33, 3x/day i.p.) and was associated with a disease-free state until experimental day 85 in $A t p 7 b^{-/-}$rat 5, corresponding to a doubling of the age when untreated LPP ${ }^{-/-}$rats become diseased.

The increasing copper burden directly affected the structural, physical, and biochemical integrity of mitochondrial membranes (Figure 2). Atp $7 b^{-/-}$liver mitochondria showed considerable matrix condensations, intermembrane space deposits, and cristae dilatations (Figure 2A). Using fluorescence polarization, we observed a steady increase in TMA-DPH polarization values in Atp $7 b^{-/-}$mitochondrial membranes and an increasing copper load (Supplemental Table 1). Thus, excessive mitochondrial copper deposition physically alters the mitochondrial membrane lipid-water interface $(33,34,39)$, plausibly by direct interaction with mitochondrial membrane proteins (18). This results in a significantly lower extent of expansion upon induction of the MPT (ref. 40 and Figure 2B). Furthermore, the capacity of Cys-A to block calcium-induced MPT was significantly impaired in $A t p 7 b^{-/-}$liver mitochondria (Figure 2C and Supplemental Table 2). Cys-A normally inhibits MPT by blocking inner mitochondrial membrane binding of its target protein Cyp-D $(40,41)$. Biochemically, Atp $7 b^{-/-}$mitochondria lost their ability to sustain their inner transmembrane potential $(\Delta \Psi)$ with a progressively greater copper load (Figure 2D and Supplemental Table 3), as well as their capacity to produce ATP (Supplemental Figure 4F). Since impaired mitochondrial membranes can seal the point of no return toward death (42), we conclude that efficient therapeutic measures against WD must rely on the reduction of mitochondrial copper concentrations.

Hepatic copper transport and homeostasis require various membrane transporters, a subset of intracellular copper chaperones (43), and low-molecular-weight binding partners like glutathione (44), but also as-yet uncharacterized intracellular ligands $(4,45)$. Copper enters the cell via high-affinity transporters like copper transporter 1 , and it is believed that differential copper affinities of the binding partners direct the metal to its final cellular destination (44). Copper can enter the $\mathrm{Cu}$-metallothionein (MT) storage pool, be routed to copper-dependent enzymes (e.g., $\mathrm{Cu} / \mathrm{Zn} \mathrm{SOD}$ or the mitochondrial cytochrome c oxidase [Cco]), or be transported out of the cell via ATP7B $(4,43)$. As the latter transporter is dysfunctional in $\mathrm{WD}$, a large part of the intracellular copper accumulates in the cytosolic MT storage pool $(46,47)$. However, in the Long-Evans Cinnamon (LEC) rat model of WD, it was shown that the cytosol from livers just before hepatitis still contained $\mathrm{Cu}$-unsaturated MTs and thus may still act as antioxidants (47). On the contrary, excess mitochondrial copper loads and severe mitochondrial destruction were observed at the onset of hepatitis in the LEC and Atp $7 b^{-/-}$rats (18), arguing for the pivotal need to reverse this mitochondrial copper overload. Given that mitochondrial $\mathrm{Cco}$, the intermembrane $\mathrm{Cu} / \mathrm{Zn}$ SOD, and the mitochondrial copper chaperones present with exceptionally low $\mathrm{Cu}^{1+}$ dissociation constants, there is a considerable driving force of copper into mitochondria $(20,44)$. Consequently, efficient mitochondrial copper removal may be achieved by compounds with high copper affinities. MB has an exceptionally high copper affinity (28) of more than $10^{-21} \mathrm{M}$, which is orders of magnitude higher than the affinities of known copper-binding proteins and ligands in eukaryotic cells (44). Thus, MB could possibly reverse mitochondrial copper overload.

In agreement with this, MB was able to efficiently deplete accumulated copper at the mitochondrial, cellular, and wholeorgan levels to a considerable extent via excretion into bile, and no significant cell toxicity or mitochondrial dysfunction was induced (Figure 3 and Table 2). Of note, we found that a higher degree of liver damage (LDH release) coincided with elevated copper mobilization upon liver perfusion with chelating agents (Supplemental Figure 3C). This finding may be of clinical relevance, as worsening of the clinical presentation is reported in up to $20 \%$ of WD patients with neurological symptoms, despite sufficiently dosed chelation treatment with D-PA or TETA $(5,48,49)$. Such deterioration may be explained by chelator-induced liver copper mobilization possibly leading to secondary copper toxicity to the brain $(48,50,51)$. Thus, in severe WD states, liver copper mobilization 
into the bloodstream may be adverse - if the low-affinity chelator used succeeds in extracting copper from hepatic stores - yet releases the bound metal before its excretion. Therefore, treating WD with high-affinity chelators such as MB, which has a copper affinity several orders of magnitude greater than that of D-PA (52), may constitute a pivotal advantage. In agreement with this notion, in preliminary studies, we observed that administering MB for 2 to 8 days (i.p.) did not elevate total brain copper levels. In addition, several studies (e.g., refs. 53, 54) reported improvement of neurological symptoms upon liver transplantation, strengthening the view that some of the excess brain copper may be excreted upon restoration of a physiological copper excretion into bile. MB causes a highly efficient excretion of copper into bile (Figure 3G). Taken together, these results support the prediction of an improvement of neurological symptoms upon MB therapy; however, this needs to be substantiated in future studies.

The superior in vitro and ex vivo de-coppering efficiency of MB led us to investigate a new treatment strategy in $A t p 7 b^{-/-}$rats. Instead of the typical WD treatment regimen involving longterm oral administration of copper chelators, we used a short but intense de-coppering treatment with repetitive injections of $\mathrm{MB}$ (Figures 4 and 5, and Table 2). In contrast to D-PA and TETA, short-term i.p. $\mathrm{MB}$ administration significantly de-coppered $A t p 7 b^{-/-}$liver mitochondria, which was paralleled by a regression of mitochondrial structural deficits and liver damage (Figure 4). In addition, following a 5-day $\mathrm{MB}$ regimen and subsequent treatment discontinuation, all animals remained healthy for at least 2 weeks (Figure 5). This delayed disease reappearance cannot be attributed to the zinc-enriched diet given from the start of MB treatment, since hepatic copper reaccumulation occurred despite zinc supplementation. In fact, in a further experiment with repeated MB treatment cycles and drug-free intervals without zinc supplementation (Table 3), the disease onset was delayed to beyond 166 days of age, an approximate doubling of the disease-free period in the $A t p 7 b^{-/-}$rat. Importantly, the disease status clearly correlated with mitochondrial, but not whole-liver, copper content (Figure 5B). Therefore, the parameters for mitochondrial copper content, structure, and biochemical functionality not only serve as early response markers for disease progression in WD patients and $A t p 7 b^{-/-}$rats (Figures 1 and 2 and Supplemental Figure $4 \mathrm{~F}$ ), but also as diagnostic biomarkers of treatment efficiency (Figure 4, C and D) and predictive markers of recurrence of liver damage (Figure 5, B and D, and Table 3). We observed that both i.p. and i.v. MB administration efficiently depleted mitochondrial copper levels (Table 2). At present, the acid sensitivity of MB (55) precludes its oral administration (in contrast to current clinical pharmacotherapy), a limitation that may, however, be overcome by using biocompatible polymers or other galenic formulations (56). Nevertheless, parenteral MB administration has fast and reliable effects, and injecting MB twice daily proved to be particularly efficient, reversing the disease phenotype even in animals that had signs of advanced liver dysfunction (Table 2 and Supplemental Figure 4, D-F). Further, concerning the temperature stability of $\mathrm{MB}$, we observed a time-dependent destabilization of $\mathrm{MB}$ at $37^{\circ} \mathrm{C}$ (Supplemental Figure 4C), which was effectively avoided, however, upon prestabilization by zinc (Supplemental Figure 4C) and did not interfere with the de-coppering properties of MB (Figure
3C and Supplemental Figure 2E). Hence, zinc-stabilized MB may be advantageous for applications requiring prolonged persistence at elevated temperatures.

With regard to drug safety, we observed no signs of MB toxicity in $A t p 7 b^{-/-}$rats treated up to 3 times per day (Table 3) or in heterozygous $A t p 7 b^{+/-}$control rats treated for 2 consecutive days (Figure 4E). However, one potential issue could be an immune response against the $\mathrm{MB}$ peptide. While it cannot be completely excluded, this appears unlikely, because small compounds like $\mathrm{MB}$, with a MW of approximately 1,000 , are considered nonimmunogenic (57). Moreover, no toxicity was observed in animals that underwent repetitive cycles of intense MB treatments (Table 3), and the first ELISA analyses testing sera of these treated animals for "anti-MB" Abs were negative. Nevertheless, this issue will need further attention.

In conclusion, we report what we believe to be an innovative therapeutic strategy for treating WD that consists of a short but intense de-coppering treatment with $\mathrm{MB}$ that proved effective, even at the acute liver failure stage in a WD rat model. In total, $24 A t p 7 b^{-/-}$rats at various stages of disease were highly effectively treated once, twice, or thrice daily, either by i.p. or i.v. injection of MB (Tables 2 and 3, and Figure 5). All treated animals were rescued, and liver damage reappeared only after several weeks of drug-free phases. The treatment of patients with bacterial siderophores is not without precedent, as exemplified by deferoxamine from Streptomyces pilosus, which is used for treating iron poisoning and hemochromatosis (58). We therefore propose MB as an additional candidate in this pharmaceutical class and as the lead drug of choice to develop a more efficient and safe treatment of WD.

\section{Methods}

\section{Patient-derived samples}

Livers from 4 WD patients with liver failure who had undergone liver transplantation at the University Hospital Heidelberg were included in this study. Two patients (patients 1 and 2) had received no prior copper chelation therapy, while 2 patients (patients 3 and 4) presented with liver failure after D-PA treatment. Upon explantation, the WD patients' livers were shock-frozen in $\mathrm{LN}_{2}$ and stored at $-80^{\circ} \mathrm{C}$. Thawed samples were immediately fixed using formalin (Bio Optica Milano) for histological analysis or glutaraldehyde (Electron Microscopy Sciences) for electron microscopic analysis.

WD patients' urinary epithelial cells were collected from freshly donated mid-stream urine.

\section{Animals}

The LPP rat strain was provided by Jimo Borjigin (University of Michigan, Ann Arbor, Michigan, USA) (16), and Lou/c rats were originally from Harlan Winkelmann. Rats were maintained on an ad libitum Altromin 1314 diet (Altromin Spezialfutter) and tap water. The basic copper content of this standard diet was $13 \mathrm{mg} / \mathrm{kg}$. Heterozygous $A t p 7 b^{+/-}$rats served as controls in this study.

\section{Animal treatments}

In vivo treatments. LPP rats were treated with MB by daily i.p. injections for 3 or 5 consecutive days or by twice-daily i.p. injections for 8 consecutive days at a dose of $150 \mathrm{mg} / \mathrm{kg} \mathrm{BW}$, or for 4 days via D-PA-contain- 
ing drinking water at a dose of $100 \mathrm{mg} / \mathrm{kg}$ BW/day or TETA-containing drinking water at $480 \mathrm{mg} / \mathrm{kg} \mathrm{BW/day} \mathrm{(assuming} 40 \mathrm{ml}$ water intake per day for a rat weighing $250 \mathrm{~g}$ ), respectively $(59,60)$. Based on a mean copper content of $250 \mu \mathrm{g} / \mathrm{g}$ wet weight (ww) in $A t p 7 b^{-/-}$rat livers at the start of treatment (18), Atp $7 b^{-/-}$rat livers of $8 \mathrm{~g}$ ww contain approximately 31.5 $\mu \mathrm{mol}$ copper. Single MB doses were chosen in amounts equimolar to this copper amount. As for D-PA, the dose and route of administration used in this study were previously reported to successfully prevent the onset of hepatitis in LEC rats in long-term regimens (59). Subchronic toxicity studies in rats have revealed no toxicity of TETA at a dose of 3,000 ppm via the drinking water (60). With respect to the mean liver copper content in 85-day-old $A t p 7 b^{-/}$rats, the molar ratios of the chelators applied was MB 1:D-PA 4.3:TETA 17.4, respectively. For i.v. MB application (150 mg/kg BW), catheters connected to a PinPort (Instech Laboratories) were inserted into the rat's femoral vein, fixed with nonabsorbable sutures, and s.c. tunneled and exteriorized through a skin incision made between the shoulders.

\section{Liver perfusion}

$A t p 7 b^{-/-}$livers (from animals aged 79-83 days) were perfused via the portal vein in a single-pass manner with Krebs-Ringer bicarbonate solution containing $5 \mathrm{mM}$ glucose, gassed with $95 \% \mathrm{O}_{2}$ and $5 \% \mathrm{CO}_{2}$, and kept at $37^{\circ} \mathrm{C}(61,62)$. The right lateral liver lobe was ligated, and its copper content served as a preperfusion control. After bile duct cannulation, a 20-minute bile sample was collected before the copper chelators were continuously added to the perfusion medium. Bile and outflow perfusates (from which LDH was measured) were collected at 10 -minute intervals $(61,62) . \mathrm{D}-\mathrm{PA} / \mathrm{HCl}(20 \mathrm{mg} / 108 \mu \mathrm{mol})$; TETA/2 $\mathrm{HCl}(20 \mathrm{mg} / 91 \mu \mathrm{mol}) ; \mathrm{TTM} / 2 \mathrm{NH}_{4}(10 \mathrm{mg} / 38 \mu \mathrm{mol}) ;$ and MB $(40 \mathrm{mg} / 35 \mu \mathrm{mol})$ were each dissolved in $50 \mathrm{ml} 0.9 \% \mathrm{NaCl}$ and continuously added to the perfusion medium via a perfusion pump (Perfusor; B. Braun Melsungen) for 2 hours. The molar ratios of the chelators used was MB 1:D-PA 3.1:TETA 2.6:TTM 1.1. Control perfusions were done with Krebs-Ringer bicarbonate solution only.

\section{Histological examination, plasma/serum AST, and bilirubin}

Formalin-fixed, paraffin-embedded liver samples were cut into $4-\mu \mathrm{m}$-thick sections and stained with either H\&E (Carl Roth GmbH + Co. KG) or Masson's trichrome (Merck KG) for analysis of fibrotic tissue. AST activity and bilirubin concentration in animal plasma or serum were measured with a Reflotron system (Roche Diagnostics).

\section{Mitochondrial analyses}

Mitochondria were derived from either frozen, explanted WD patients' livers or freshly prepared rat liver homogenates, as described previously $(35,63)$, and purified by differential and density gradient centrifugation using either Percoll (GE Healthcare Europe) or Nycodenz (Axis-Shield). Fresh rat liver mitochondria were used for respiratory measurements, chelator treatments, analyses of swelling (MPT), transmembrane potential $\left(\Delta \psi_{\mathrm{m}}\right)$, polarization experiments, and ATP synthesis and were fixed with glutaraldehyde for subsequent electron microscopic analyses. Stored frozen mitochondria were used for respiratory complex IV activity and metal analyses.

Mitochondrial functional integrity was assessed by standard respiratory measurements in a Clark-type oxygen electrode (Oxygraph; Hansatech Instruments) (18). ATP synthesis was analyzed by the ATP Bioluminescence Assay Kit (Roche) (18). Mitochondrial swelling was measured by light scattering at $540 \mathrm{~nm}$, and $\Delta \psi_{\mathrm{m}}$ was followed by Rh123 fluorescence quenching in a 96-well plate reader (BioTek, Synergy 2; Bad Friedrichshall) (64). For calculation, in cases with onset but not-yet finished MMP depletion, end values were set to 180 minutes. Polarization was measured in DPH and TMA-DPH-dyed mitochondria $(33,34)$. In brief, mitochondria $(3 \mathrm{mg} / \mathrm{ml})$ were incubated for $30 \mathrm{~min}$ utes at $37^{\circ} \mathrm{C}$ with either DPH or TMA-DPH $(50 \mu \mathrm{M}$ and $20 \mu \mathrm{M}$, respectively). Parallel and perpendicular fluorescence was assessed in duplicate at ex $366 \mathrm{~nm}$ and em $425 \mathrm{~nm}$. Polarization was calculated (65) in mPol using the following formula: $P=\left(I_{\|}-G \times I_{\perp}\right) /\left(I_{\|}+G \times I_{\perp}\right) ; G=0.89$.

In vitro treatment of isolated mitochondria with chelators. Freshly isolated $A t p 7 b^{-1-}$ mitochondria with elevated copper levels were subjected to 30-minute chelator treatments with either $2 \mathrm{mM} \mathrm{D}-\mathrm{PA}$, TETA, TTM, or MB and subsequently repurified by a Nycodenz-gradient to separate liberated copper from mitochondria. For validation, mitochondria from $A t p 7 b^{+/-}$control rats were incubated with $1 \mathrm{mM}$ DTT (Roche) for 5 minutes at room temperature, and thereafter, $\mathrm{Cu}^{2+}$ (Copper (II) Chloride Dihydrate; Sigma-Aldrich) was added at final concentrations of 200-600 $\mu \mathrm{M}$ for 20 minutes. Copper-loaded mitochondria were then repurified by Nycodenz-gradient centrifugation and subsequently treated with chelators as described above.

\section{Cell culture}

All cell lines were from ATCC unless otherwise indicated.

HepG2 cells were kept in MEM (Life Technologies) with 2\% FCS (Biochrom). We found that $\mathrm{Zn}-\mathrm{MB}$ was time stable at $37^{\circ} \mathrm{C}$, in contrast to metal-free MB (Supplemental Figure $4 \mathrm{C}$ ). Therefore, Zn-MB, generated by preparing a $20-\mathrm{mM} \mathrm{MB}$ solution and adding an equimolar concentration of $\mathrm{Zn}^{2+}$ (Zinc acetate; Sigma-Aldrich) solution under $\mathrm{pH}$ control, was used in cell culture experiments.

A neutral red cell toxicity assay (Sigma-Aldrich) was performed as described elsewhere (66). HepG2 cells $\left(2 \times 10^{4}\right)$ were incubated for 24 hours with medium alone (negative control), 0.05 to $1 \mathrm{mM}$ zinc-MB or TTM, 0.002-1 mM copper-histidine, or $0.25 \mathrm{mM}$ carbonyl cyanide m-chlorophenylhydrazone (CCCP) as a mitochondriotoxic positive control and were subsequently analyzed.

For immunofluorescence staining, $2 \times 10^{4}$ HepG 2 cells were incubated with medium alone, $500 \mu \mathrm{M} \mathrm{MB}$, or $250 \mu \mathrm{M}$ CCCP in black 96-well plates with a clear glass bottom (PerkinElmer). Staining was done using $1.6 \mu \mathrm{M}$ Hoechst 33342 (excitation wavelength [ex] 360-400 nm; emission wavelength [em] 410-480 nm); $300 \mathrm{nM}$ MitoTracker Deep Red (ex 620-640 nm, em 650-760 nm); and $1 \mu \mathrm{M}$ nonyl acridine orange (NAO) (ex 460-490 nm, em 500-550 nm) for 40 minutes at $37^{\circ} \mathrm{C}$. After a washing step, fluorescence was analyzed using an Operetta System (PerkinElmer).

Cellular de-coppering by MB was assessed in HepG2 cells pretreated for 24 hours with either medium or $15 \mu \mathrm{M}$ copper-histidine and after a subsequent 24-hour treatment with $500 \mu \mathrm{M}$ MB. After 2 washing steps, copper levels in $2.5 \times 10^{6} \mathrm{HepG} 2$ cells were determined by inductively coupled plasma optical emission spectrometry (ICPOES). Cellular MB uptake was determined from HepG2 cell lysates incubated for 2 or 24 hours with MB at different concentrations by a competitive ELISA using an anti-MB mAb.

\section{MB Ab generation and competitive ELISA}

$\mathrm{Lou} / \mathrm{c}$ rats were immunized with a mixture of OVA-coupled MB $(50 \mu \mathrm{g})$ (Squarix), 5 nmol CPG oligonucleotide (TIB MOLBIOL; Syntheselabor), 
$500 \mu \mathrm{lBS}$, and $500 \mu \mathrm{l}$ incomplete Freund's adjuvant (Sigma-Aldrich). A boost without adjuvant was given 6 weeks after the primary injection. Fusion was performed using rat spleen and P3X63Ag8.653 myeloma (ATCC) cells (67). mAbs from these tissue culture supernatants (TCSs) were tested in an ELISA-plate solid-phase immunoassay (Corning Life Sciences) against BSA-coupled MB or an irrelevant BSA-coupled peptide at a concentration of $4 \mu \mathrm{g} / \mathrm{ml}$. For detection, HRP-conjugated mAbs with IgG-specific isotypes were purified from myeloma supernatants (TIB173: IgG2a; TIB174: IgG2b; and TIB170: IgG1, all from ATCC; R-2c: IgG2c, from Helmholtz Center Munich) using the Protein A Sepharose 4 FastFlow (GE Healthcare Europe), and HRP conjugated using the LYNX Rapid HRP Antibody Conjugation Kit (Bio-Rad AbD Serotec). HRP was visualized with the 1-Step Ultra TMB-ELISA (Thermo Fisher Scientific). Eighty-six hybridomas that reacted specifically with MB were frozen, and $\mathrm{Ab}$-containing TCSs were used for further analysis.

Competitive ELISA. Fifty microliters of each TCS (diluted 1:10 with buffer consisting of PBS, $5 \%$ FCS, and $0.01 \%$ sodium azide) was preincubated with $50 \mu \mathrm{l} \mathrm{MB}$ solution (1,000 $\mathrm{ng} / \mathrm{ml}$ in buffer) or with buffer overnight (in duplicate). For screening, $50 \mu$ of these samples was added for 10 minutes to MB-coated ELISA plates. After washing, bound Abs against $\mathrm{MB}$ were detected with HRP-conjugated mAbs as described above. $\mathrm{mAbs}$ that recognized free $\mathrm{MB}$ (no signal when preincubated with $\mathrm{MB}$, positive signal in the buffer control) were further analyzed in serial MB dilutions (1,000 ng/ml to $2 \mathrm{ng} / \mathrm{ml})$. The $4 \mathrm{mAbs}$ that best recognized free MB were established (10B10, 12D9, 18H7, and 21G5, all of the rat IgG2a subclass). In subsequent titration experiments on MB-coated ELISA plates, the titer of $10 \mathrm{~B} 10$ was found to be of highest reactivity and was therefore subsequently used (1:500 dilution, 1-hour preincubation).

\section{Generation of HLCs from WD patients}

WD patients' urinary epithelial cells were pelleted at $400 \times g$ for 10 minutes from freshly donated mid-stream urine (68). Cells were cultured in culture medium (DMEM/F12; Lonza Group) supplemented with 10\% FBS (PAA Laboratories); $0.1 \mathrm{mM}$ nonessential aa (NEAA) (Sigma-Aldrich); $0.1 \mathrm{mM} \beta$-mercaptoethanol (Gibco); $1 \mathrm{mM}$ GlutaMAX (Life Technologies); and SingleQuot Kit CC-4127 REGM (Lonza Group) and reprogrammed into iPSCs by nucleofection of episomal expression vectors pCXLE-hOCT3/4-shp53-F, pCXLE-hSK, and pCXLE-hUL (Addgene) using the Amaxa Basic Nucleofector Kit (Lonza Group AG; VPI-1005). iPSCs were maintained on Matrigel-coated plates (Corning) in mTeSR cell culture medium and dissociated with 1 $\mathrm{U} / \mathrm{ml}$ dispase (STEMCELL Technologies) into small clusters and subcultured every 5 to 7 days. WD iPSCs were differentiated into HLCs by a modification of the method reported previously $(69,70)$. iPSCs $(5$ $\left.\times 10^{4}\right)$ in Matrigel-precoated 6-well plates were incubated in DMEM/ F12 enriched with $100 \mathrm{ng} / \mathrm{ml}$ recombinant Activin-A (PeproTech); $100 \mathrm{ng} / \mathrm{ml} \mathrm{FGF} 2$ (PeproTech); and $50 \mathrm{ng} / \mathrm{ml}$ recombinant human WNT3A (R\&D Systems). Subsequently, medium was changed up to day 14 according to standard protocols $(69,70)$. Cells were characterized by flow cytometry to assess albumin expression using the anti-human serum albumin Ab (Abcam; catalog ab2406) and the goat anti-rabbit IgG $(\mathrm{H}+\mathrm{L})$ secondary Ab with Alexa Fluor 594 conjugate (Thermo Fisher Scientific; catalog A-11012) for detection and by quantitative real-time PCR to assess typical markers of hepatocyte lineage.

HLCs were incubated on day 14 with copper-histidine $(15 \mu \mathrm{M})$ for 24 hours in a 6-well plate. The following day, medium was removed and changed to OptiMEM (Gibco) containing MB (300 $\mu \mathrm{M})$. After a 24-hour incubation, washed cells were collected and counted and their copper content assessed.

\section{Metal content determination}

Copper levels in liver homogenates, cell lysates, and mitochondrial preparations were analyzed by ICP-OES (Ciros Vision; SPECTRO Analytical Instruments) after wet-ashing of samples with $65 \%$ nitric acid (Merck KGaA) (18).

\section{Electron microscopic analysis}

Electron microscopic analysis of liver tissues and mitochondria was done as previously described (35).

\section{Mitochondria structural analyses}

For structural analyses, isolated mitochondria were categorized as type 1 (normal structured mitochondria of the "condensed" type; ref. 71); type 2 (mitochondria with minor alterations such as slightly increased cristae); type 3 (mitochondria with massively increased cristae); and type 4 (mitochondria with massive matrix condensations, matrix vacuolization, detachments of the inner boundary membrane, and severe cristae deformations).

\section{Miscellaneous}

MB was isolated from the spent media of Methylosinus trichosporium OB3b as previously described (72). Endotoxin in MB was detected by a kinetic chromogenic method (Charles River Laboratories) and was on average $4.5 \mathrm{IU} / \mathrm{mg}$. Copper-histidine solutions (1:3) were prepared as described previously (73). Protein quantification was done by the Bradford assay. Cytochrome $\mathrm{C}$ oxidase activity in isolated mitochondria was determined as described elsewhere (74).

\section{Chemicals}

D-PA was a gift of Heyl Pharma; TETA was from Sigma-Aldrich; and TTM (98\% pure) was a gift of KT. Suzuki (Chiba University, Chiba Japan). CCCP was obtained from Sigma-Aldrich; DPH, TMA-DPH, MitoTracker Deep red, and NAO were obtained from Molecular Probes; and Hoechst 33342 was obtained from Life Technologies.

\section{Statistics}

Throughout this study " $\mathrm{N}$ " equals the number of analyzed animals and " $n$ " the number of measurements. Data are presented as the mean \pm SD. A Grubbs outlier test (ESD method, extreme studentized deviate) was performed if more than 4 technical replicates were available. A significance level of $\alpha=0.05$ was used. In detail, data from Figure 1C, Figure 2, A and B, Supplemental Figure 4F, and Supplemental Tables 1-3 were corrected for outliers. Statistical significance was analyzed using 1-way ANOVA with Tukey's multiple comparisons test when comparing 3 or more sample sets (GraphPad Prism 7; GraphPad Software). For comparison between 2 groups the unpaired 2-tailed Student's $t$ test with Welch's correction was used (GraphPad Prism 7). A $P$ value of less than 0.05 was considered statistically significant.

\section{Study approval}

All patients provided informed consent, and the study was approved by the ethics committee of the Medical University of Heidelberg, Germany (for the study of liver samples from patients with WD) and by the 
Ethik-Kommission der Ärztekammer Westfalen-Lippe und der Westfälischen Wilhelms-Universität Münster of Münster, Germany (for the study of WD patients' urinary epithelial cells).

Animal experiments were approved by the government authorities of the Regierung von Oberbayern, Munich, Germany, and all animals were treated according to the guidelines for the care and use of laboratory animals of the Helmholtz Center Munich.

\section{Author contributions}

JL and CL designed and performed experiments, analyzed data, and wrote the manuscript. RW, S. Schmitt, S. Schulz, CE, TR, and YK performed experiments. DJ, FN, BKS, and PS performed histochemical and transmission electron microscopic analyses. AADS, NB, and BSB produced MB samples. AF and EK established primary anti-MB Abs and performed ELISA analyses. GD, FPR, SH, FES, NAD, and JA analyzed data. VS, CN, and HHJS constructed and performed HLC experiments. UM and DNG examined patients and collected clinical samples. GK designed experiments and wrote the manuscript. KHW examined patients, collected clinical samples, and designed experiments. HZ designed experiments, analyzed data, wrote the manuscript, and directed this study.

\section{Acknowledgments}

We are deeply indebted to the late Friedericke Eckardt-Schupp, who continuously supported this work. The authors would like to thank E. Samson for technical assistance and M. Atkinson and E.E. Rojo for critical reading of the manuscript. This study was supported in part by the Deutsche Forschungsgemeinschaft (DFG) grant RU742/6-1 (to H. Zischka) and STR 1160/1-2 (to B.K. Straub).

Address correspondence to: Hans Zischka, Institute of Molecular Toxicology and Pharmacology, Helmholtz Center Munich, German Research Center for Environmental Health, Ingolstaedter Landstrasse 1, 85764 Munich-Neuherberg, Germany. Phone: 49.89.3187.2663; E-mail: zischka@helmholtz-muenchen.de.
1. Gitlin JD. Wilson disease. Gastroenterology. 2003;125(6):1868-1877.

2. Tanzi RE, et al. The Wilson disease gene is a copper transporting ATPase with homology to the Menkes disease gene. Nat Genet. 1993;5(4):344-350.

3. Bull PC, Thomas GR, Rommens JM, Forbes JR, Cox DW. The Wilson disease gene is a putative copper transporting P-type ATPase similar to the Menkes gene. Nat Genet. 1993;5(4):327-337.

4. Kim BE, Nevitt T, Thiele DJ. Mechanisms for copper acquisition, distribution and regulation. Nat Chem Biol. 2008;4(3):176-185.

5 . Weiss KH, Stremmel W. Evolving perspectives in Wilson disease: diagnosis, treatment and monitoring. Curr Gastroenterol Rep. 2012;14(1):1-7.

6. Roberts EA, Sarkar B. Liver as a key organ in the supply, storage, and excretion of copper. Am J Clin Nutr. 2008;88(3):851S-854S.

7. Roberts EA, Schilsky ML, American Association for Study of Liver Diseases. Diagnosis and treatment of Wilson disease: an update. Hepatology. 2008;47(6):2089-2111.

8. European Association for Study of Liver. EASL Clinical Practice Guidelines: Wilson's disease. J Hepatol. 2012;56(3):671-685.

9. Schilsky ML. Treatment of Wilson's disease: what are the relative roles of penicillamine, trientine, and zinc supplementation? Curr Gastroenterol Rep. 2001;3(1):54-59.

10. Schilsky ML. Liver transplantation for Wilson's disease. Ann N Y Acad Sci. 2014;1315:45-49.

11. Walshe JM. Cause of death in Wilson disease. Mov Disord. 2007;22(15):2216-2220.

12. Murillo O, et al. Long-term metabolic correction of Wilson's disease in a murine model by gene therapy. J Hepatol. 2016;64(2):419-426.

13. Roy-Chowdhury J, Schilsky ML. Gene therapy of Wilson disease: A "golden" opportunity using rAAV on the 50th anniversary of the discovery of the virus. J Hepatol. 2016;64(2):265-267.

14. Spincemaille $\mathrm{P}$, et al. The plant decapeptide OSIP108 prevents copper-induced apoptosis in yeast and human cells. Biochim Biophys Acta. 2014;1843(6):1207-1215.
15. Pujol AM, et al. Hepatocyte targeting and intracellular copper chelation by a thiol-containing glycocyclopeptide. JAm Chem Soc. 2011;133(2):286-296.

16. Ahmed S, Deng J, Borjigin J. A new strain of rat for functional analysis of PINA. Brain Res Mol Brain Res. 2005;137(1-2):63-69.

17. Burkhead JL, Gray LW, Lutsenko S. Systems biology approach to Wilson's disease. Biometals. 2011;24(3):455-466.

18. Zischka $\mathrm{H}$, et al. Liver mitochondrial membrane crosslinking and destruction in a rat model of Wilson disease. JClin Invest. 2011;121(4):1508-1518.

19. Sternlieb I. Mitochondrial and fatty changes in hepatocytes of patients with Wilson's disease. Gastroenterology. 1968;55(3):354-367.

20. Zischka H, Lichtmannegger J. Pathological mitochondrial copper overload in livers of Wilson's disease patients and related animal models. Ann N Y Acad Sci. 2014;1315:6-15.

21. Roberts EA, Robinson BH, Yang S. Mitochondria structure and function in the untreated Jackson toxic milk (tx-j) mouse, a model for Wilson disease. Mol Genet Metab. 2008;93(1):54-65.

22. Huster D, et al. Consequences of copper accumulation in the livers of the Atp7 $\mathrm{b}^{-/-}$(Wilson disease gene) knockout mice. Am JPathol. 2006;168(2):423-434.

23. Choi DW, et al. Spectral, kinetic, and thermodynamic properties of $\mathrm{Cu}(\mathrm{I})$ and $\mathrm{Cu}(\mathrm{II})$ binding by methanobactin from Methylosinus trichosporium OB3b. Biochemistry. 2006;45(5):1442-1453.

24. El Ghazouani A, et al. Copper-binding properties and structures of methanobactins from Methylosinus trichosporium OB3b. Inorg Chem. 2011;50(4):1378-1391.

25. Kim HJ, et al. Methanobactin, a copper-acquisition compound from methane-oxidizing bacteria. Science. 2004;305(5690):1612-1615.

26. Behling LA, et al. NMR, mass spectrometry and chemical evidence reveal a different chemical structure for methanobactin that contains oxazolone rings. J Am Chem Soc. 2008;130(38):12604-12605.
27. Semrau JD, DiSpirito AA, Yoon S. Methanotrophs and copper. FEMS Microbiol Rev. 2010;34(4):496-531.

28. Semrau JD, et al. Methanobactin MmoD work in concert to act as the 'copper-switch' in methanotrophs. Environ Microbiol. 2013;15(11):3077-3086.

29. Gu W, et al. A TonB-dependent transporter is responsible for methanobactin uptake by Methylosinus trichosporium OB3b. Appl Environ Microbiol. 2016;82(6):1917-1923.

30. Vita N, et al. A four-helix bundle stores copper for methane oxidation. Nature. 2015;525(7567):140-143.

31. Das SK, Ray K. Wilson's disease: an update. Nat Clin Pract Neurol. 2006;2(9):482-493.

32. Ferenci P. Phenotype-genotype correlations in patients with Wilson's disease. Ann N Y Acad Sci. 2014;1315:1-5.

33. Prendergast FG, Haugland RP, Callahan PJ. 1-[4-(Trimethylamino)phenyl]-6-phenylhexa1,3,5-triene: synthesis, fluorescence properties, and use as a fluorescence probe of lipid bilayers. Biochemistry. 1981;20(26):7333-7338.

34. Duportail G, Weinreb A. Photochemical changes of fluorescent probes in membranes and their effect on the observed fluorescence anisotropy values. Biochim Biophys Acta. 1983;736(2):171-177.

35. Zischka H, et al. Electrophoretic analysis of the mitochondrial outer membrane rupture induced by permeability transition. Anal Chem. 2008;80(13):5051-5058.

36. Gitlin JD. Copper homeostasis: specialized functions of the late secretory pathway. Dev Cell. 2014;29(6):631-632.

37. Ogra Y, Miyayama T, Anan Y. Effect of glutathione depletion on removal of copper from LEC rat livers by tetrathiomolybdate. J Inorg Biochem. 2010;104(8):858-862.

38. Gu M, et al. Oxidative-phosphorylation defects in liver of patients with Wilson's disease. Lancet. 2000;356(9228):469-474.

39. Ricchelli F, Gobbo S, Moreno G, Salet C. Changes of the fluidity of mitochondrial membranes induced by the permeability transition. Biochem- 
istry. 1999;38(29):9295-9300.

40. Halestrap AP. A pore way to die: the role of mitochondria in reperfusion injury and cardioprotection. Biochem Soc Trans. 2010;38(4):841-860.

41. Schneider MD. Cyclophilin D: knocking on death's door. Sci STKE. 2005;2005(287):pe26.

42. Green DR, Kroemer G. The pathophysiology of mitochondrial cell death. Science. 2004;305(5684):626-629.

43. Zheng W, Monnot AD. Regulation of brain iron and copper homeostasis by brain barrier systems implication in neurodegenerative diseases. Pharmacol Ther. 2012;133(2):177-188.

44. Banci L, Bertini I, Ciofi-Baffoni S, Kozyreva T, Zovo K, Palumaa P. Affinity gradients drive copper to cellular destinations. Nature. 2010;465(7298):645-648.

45. Leary SC, Winge DR, Cobine PA. "Pulling the plug" on cellular copper: the role of mitochondria in copper export. Biochim Biophys Acta. 2009;1793(1):146-153.

46. Klein D, Bartsch R, Summer KH. Quantitation of $\mathrm{Cu}$-containing metallothionein by a Cd-saturation method. Anal Biochem. 1990;189(1):35-39.

47. Shishido N, Nakayama K, Takazawa A, Ohyama T, Nakamura M. Cu-metallothioneins (Cu(I) 8MTs) in LEC rat livers 13 weeks after birth still act as antioxidants. Arch Biochem Biophys. 2001;387(2):216-222.

48. Weiss KH, Stremmel W. Clinical considerations for an effective medical therapy in Wilson's disease. Ann N Y Acad Sci. 2014;1315:81-85.

49. Walshe JM, Yealland M. Chelation treatment of neurological Wilson's disease. The Quarterly Journal of Medicine. 1993;86(3):197-204.

50. Brewer GJ, Terry CA, Aisen AM, Hill GM. Worsening of neurologic syndrome in patients with Wilson's disease with initial penicillamine therapy. Arch Neurol. 1987;44(5):490-493.

51. Chen DB, et al. Penicillamine increases free copper and enhances oxidative stress in the brain of toxic milk mice. PLoS One. 2012;7(5):e37709.

52. Klein D, Lichtmannegger J, Heinzmann U, Summer KH. Dissolution of copper-rich granules in hepatic lysosomes by D-penicillamine prevents the development of fulminant hepatitis in Long-Evans cinnamon rats. J Hepatol. 2000;32(2):193-201.

53. Weiss KH, et al. Outcome and development of symptoms after orthotopic liver transplantation for Wilson disease. Clin Transplant. 2013;27(6):914-922.

54. Schumacher G, et al. Liver transplantation in neurologic Wilson's disease. Transplant Proc. 2001;33(1-2):1518-1519.

55. Kim HJ, Galeva N, Larive CK, Alterman M, Graham DW. Purification and physical-chemical properties of methanobactin: a chalkophore from Methylosinus trichosporium OB3b. Biochemistry. 2005;44(13):5140-5148.

56. Park JW, et al. Multifunctional delivery systems for advanced oral uptake of peptide/protein drugs. Curr Pharm Des. 2015;21(22):3097-3110.

57. Trier NH, Hansen PR, Houen G. Production and characterization of peptide antibodies. Methods. 2012;56(2):136-144.

58. Cappellini MD, Musallam KM, Taher AT. Overview of iron chelation therapy with desferrioxamine and deferiprone. Hemoglobin. 2009;33(suppl 1):S58-S69.

59. Togashi Y, et al. D-penicillamine prevents the development of hepatitis in Long-Evans Cinnamon rats with abnormal copper metabolism. Hepatology. 1992;15(1):82-87.

60. Greenman DL, et al. Subchronic toxicity of triethylenetetramine dihydrochloride in $\mathrm{B} 6 \mathrm{C} 3 \mathrm{~F} 1$ mice and F344 rats. Fundam Appl Toxicol. 1996;29(2):185-193.

61. Beuers U, et al. Taurolithocholic acid exerts cholestatic effects via phosphatidylinositol 3-kinase-dependent mechanisms in perfused rat livers and rat hepatocyte couplets. J Biol Chem. 2003;278(20):17810-17818.

62. Beuers U, et al. Tauroursodeoxycholic acid inserts the apical conjugate export pump, Mrp2, into canalicular membranes and stimulates organic anion secretion by protein kinase Cdependent mechanisms in cholestatic rat liver. Hepatology. 2001;33(5):1206-1216.
63. Schmitt S, et al. A semi-automated method for isolating functionally intact mitochondria from cultured cells and tissue biopsies. Anal Biochem. 2013;443(1):66-74.

64. Schulz S, et al. Progressive stages of mitochondrial destruction caused by cell toxic bile salts. Biochim Biophys Acta. 2013;1828(9):2121-2133.

65. Grebowski J, Krokosz A, Puchala M. Membrane fluidity and activity of membrane ATPases in human erythrocytes under the influence of polyhydroxylated fullerene. Biochim Biophys Acta. 2013;1828(2):241-248.

66. Repetto G, del Peso A, Zurita JL. Neutral red uptake assay for the estimation of cell viability/ cytotoxicity. Nat Protoc. 2008;3(7):1125-1131.

67. Kohler G, Milstein C. Continuous cultures of fused cells secreting antibody of predefined specificity. Nature. 1975;256(5517):495-497.

68. Zhou T, et al. Generation of induced pluripotent stem cells from urine. J Am Soc Nephrol. 2011;22(7):1221-1228.

69. Basma H, et al. Differentiation and transplantation of human embryonic stem cell-derived hepatocytes. Gastroenterology. 2009;136(3):990-999.

70. Chen Y, et al. Amelioration of Hyperbilirubinemia in Gunn Rats after transplantation of human induced pluripotent stem cell-derived hepatocytes. Stem Cell Rep. 2015;5(1):22-30.

71. Hackenbrock CR. Ultrastructural bases for metabolically linked mechanical activity in mitochondria. II. Electron transport-linked ultrastructural transformations in mitochondria. JCell Biol. 1968;37(2):345-369.

72. Bandow NL, et al. Isolation of methanobactin from the spent media of methane-oxidizing bacteria. Methods Enzymol. 2011;495:259-269.

73. Hoppe-Tichy T, Nguyen TH, Hentze BW, Lorke M. [Manufacturing and stability of copper-histidine solution for treatment of Menkes' Kinky Hair Syndrome]. Die Pharmazie. 2005;60(3):205-207.

74. Kiebish MA, et al. Lipidomic analysis and electron transport chain activities in C57BL/6 mouse brain mitochondria. J Neurochem. 2008;106(1):299-312. 